
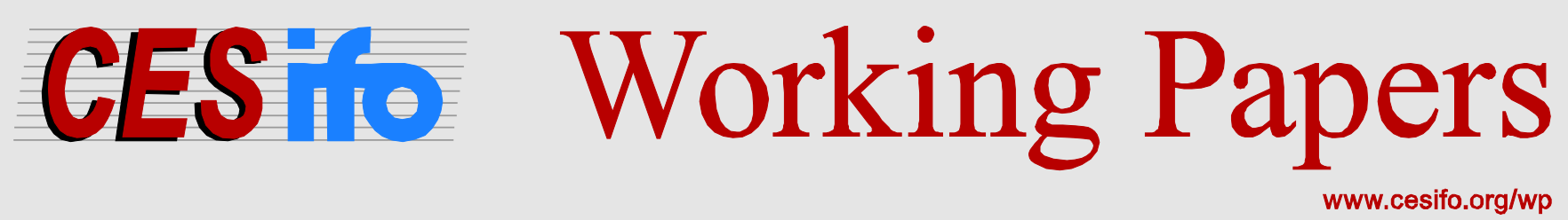

\title{
A Signaling Model of University Selection
}

\author{
Ivan Anić \\ Vladimir Božin \\ Branko Urošević
}

CESIFO WORKING PAPER NO. 5741

CATEGORY 5: ECONOMICS OF EDUCATION

FEBRUARY 2016

An electronic version of the paper may be downloaded

- from the SSRN website:

- from the RePEc website:

- from the CESifo website: WwW.SSRN.com

Www.RePEc.org

www.CESifo-group.org/wp 


\title{
A Signaling Model of University Selection
}

\begin{abstract}
We propose a signaling model of student enrollment dynamics based on probabilities of completion of studies, under different rationality assumptions. There are two types of students and two schools. School of graduation serves as a signal of student productivity to prospective employers. The benchmark case is when both students and employers are rational. We, then, relax rationality assumptions and show that, while intermediate dynamics becomes more complex, possible long run equilibria stay essentially the same. Importantly, comparative statics can be quite surprising and differ significantly from the full rationality case.
\end{abstract}

JEL-codes: I200, J240, D820, D830, D840.

Keywords: job market signaling, university selection, enrolment dynamics, dropout rate.

Ivan Anić

University of Belgrade

Faculty of Mathematics

Studentski trg 16

Serbia - 11000 Belgrade

ianic@matf.bg.ac.rs
Vladimir Božin

University of Belgrade

Faculty of Mathematics

Studentski trg 16

Serbia - 11000 Belgrade

bozin@matf.bg.ac.rs

\author{
Branko Urošević \\ University of Belgrade \\ Faculty of Economics \\ Kamenička 6 \\ Serbia - 11000 Belgrade \\ urosevic@ekof.bg.ac.rs
}

Views expressed in this paper do not necessarily reflect the views of the authors' respective institutions. The authors would like to thank Gerhard Illing, Boyan Jovanovich, Manfred Holler, Carsten Sprenger, Paul Schweinzer, and participants of the seminars at the National Bank of Serbia, University of Bath, University of Kent, Ludwig Maximilian University Munich, and ICEF, Higher School of Economics, Moscow for useful comments. All remaining errors are solely our own. B.U and I.A. gratefully acknowledge support of the Serbian Ministry for Education, Science and Technology Grant No. 179005. 


\section{Introduction and literature review}

In this paper we study dynamics of university selection by prospective students. There are many reasons why students may choose to go to a particular school or university. One reason could be to signal their quality to prospective employers (Spence, 1973, 1974), to acquire new skills (Altonji, 1993; Alos-Ferrer and Prat, 2013), to achieve higher social status (Akerlof, 1997) or to network (Calvo-Armengol et al., 2009). Arguably, an important reason to select a particular school is students' expectation of their postgraduation salary. In a recent empirical article, Broecke (2012), for instance, finds that students may apply more readily to universities that are more difficult to enter into because of a premium in post-graduation salary (for the UK, at least). While wage premium related to pre-entrance school selectivity is studied by many ${ }^{3}$, to date little is known about how relative likelihood of graduation (i.e. probability of graduating from different schools) and different ways in which students and employers form their expectations influence university selection process by students. We present a theoretical framework to address these issues.

Our paper follows the seminal work of Spence $(1973,1974)$ by assuming that schooling can add value through signaling even if it does not enhance productivity. ${ }^{4}$ In our model there are two types of students, those with high and those with low productivity. They select between two schools. Cost of education is modeled implicitly through probability of not completing the selected school. Probabilities of completion depend on school and student types.

Empirical evidence suggests that education has stronger signaling impact in the initial stages of one's career which may or may not persist in the long run: while Farber and Gibbons (1996), for example, find high and persistent signaling value of education, Altonji and Pierret (2001) do not. ${ }^{5}$ Thus, we focus on market for new graduates. ${ }^{6}$

Universities in our model are not active decision makers. In particular, they do not change curriculum in order to adjust to competitive pressure. While this is clearly an oversimplification of reality, it does reflect a remarkable rigidity of university curriculae often observed practice. In addition, they do not screen students at the enrollment stage but, rather, through differentiation in the rates of completion. This reflects practices at many European and South American universities.

\footnotetext{
${ }^{3}$ See, e.g. Broeke (2012) for a review of that literature.

4 This assumption is quite common in the signaling literature on education (see Riley (2001) for a comprehensive summary).

${ }^{5}$ On theoretical grounds, Jovanovic (1979) shows that on-the-job training limits the predictive value of education level on worker wages to just a few years after the initial employment.

${ }^{6}$ Weiss (1983) shows that if worker type separation is achieved already at the beginning of their schooling process, then workers may be offered jobs before they complete their course of studies. Swinkels (1999) proposes a model where it may be optimal for workers to complete just part of their studies.
} 
Students select schools based on their expectations about their future salary. There is an important and burgeoning literature on this issue. The results are, unfortunately, inconclusive. While Wiswall and Zafar (2011), for example, find that the majority of students in their experiment do not use rational (Bayesian) updating about their future salary prospects, Zafar (2011) finds the opposite result. For this reason, we consider two ways in which expectations of students are formed: rational (see Section 3) and naïve (Sections 4 and 5). ${ }^{7}$ With rational expectations, students are forward looking. They know the behavior of employers and take it into the account when assessing post-graduation salary level. With naïve expectations, in contrast, students are backward looking. They take last available market salary for school graduates as the best estimate of the salary offered to them upon graduation.

Perfectly competitive employers set wages based on their expectations about student productivity. They use rational (Section 3 and 4) or adaptive (Section 5) expectations. In the former case, employers are forward looking while in the latter case, they use past reputation of schools as a guide.

We consider enrollment dynamics in three cases: a benchmark case when both students and employers use rational expectations (RR), an intermediate case in which students use naïve expectations while employers are rational $(\mathrm{RN})$, and the case in which both students and employers are backward looking so that students use naïve while employers use adaptive expectations (AN). In particular, we study how likelihood of graduation from the two schools by each of student types influences equilibrium enrollment dynamics and to what extent that dynamics depends on ways expectations are formed.

We characterize possible enrollment dynamics and long-run equilibria for each of these cases and find conditions under which they obtain. Long-run equilibria are quite similar and can be either pooling, separating or a cycle (mixed in RR case). On the other hand, reduction of rationality typically increases the number of steps (generations of students) needed to reach long run outcome. Importantly, departure from full rationality may lead to quite surprising comparative statics results. For example, a small increase in likelihood of graduation of high productivity students at the more challenging school, ceteris paribus, under certain circumstances may lead to the loss of all students at that school. This could not happen in RR case.

To the best of our knowledge, this is the first theoretical paper that studies how probability of completing studies impacts dynamics of university selection in both fully rational and less than fully rational settings. While signaling literature is vast, the closest to ours are three papers. Noldeke and Samuelson (1997) study a dynamic signaling model in a different setting, namely, in a market with multiple sellers and one buyer. They

\footnotetext{
${ }^{7}$ Actual student expectation formation may be much more complicated than simple assumptions that we make (see Manski (1989) and Manski (1993), among others).
} 
derive dynamic equilibria similar to our RN case. They propose to study effects of adaptive expectations of the buyer as a direction of further research. We address this issue in depth, albeit in a somewhat different setup (Section 5). Amaya (2009) studies signaling problem in a setting similar to our RR approach (Section 3) and characterizes corresponding equilibrium conditions. Like us, Altonji (1993) incorporates probability of completion into a dynamic educational model. However, that paper focuses on sequential decisions of single individual while we study decisions of different student cohorts across time.

The paper is structured as follows. In Section 2 we introduce the basic model and notation. Rational expectation equilibrium is studied in Section 3. Section 4 analyzes a dynamic equilibrium with rational employers and naïve students. Section 5 extends the analysis to the situation where employers use adaptive expectations while students use naïve ones. Section 6 considers some interesting examples and comparative statics. Section 7 concludes. Proofs and more technical points are relegated to the Appendix.

\section{The Model}

We study enrollment decisions by prospective students. Following Spence (1973), schooling is assumed not to change the productivity of students. Rather, it serves purely as a signal of student quality to prospective employer. ${ }^{8}$ There are two types of students, those with high and those with low level of productivity. These levels are denoted, respectively, as $S_{H}$ and $S_{L}$, where $S_{H}>S_{L}$.

Students can choose between two schools (universities), $U_{1}$ and $U_{2}$. Selecting a different school may provide different signal to prospective employers. The first school is more challenging, and, thus, more difficult to complete for both types of students. At the same time, for a given school, high productivity students are more likely to complete the course of studies than low productivity ones. ${ }^{9}$ All agents (students and employers) are assumed to be risk neutral.

Let us denote the fractions of high and low productivity students that complete $U_{i}$ by $p_{H i}, p_{L i}, i=1,2$ and assume that they are constant in time ${ }^{10}$. Furthermore, we assume

\footnotetext{
${ }^{8}$ This is assumed for simplicity and to be consistent with the standard signaling argument. In reality, schooling is likely to positively influence productivity.

${ }^{9}$ For simplicity, we assume that schools are passive, i.e. they set their curricula and grading criterions once and for all. In addition, they accept all students that apply, thus giving students the power of choice. Instead of discriminating at the entrance between high and low productivity students, schools do so during the course of studies by differential likelihood of graduation by the two student types. Such behavior is common in many European and Latin American countries.

${ }^{10}$ For simplicity, we treat the number of students that complete each university as a continuous variable. Also, students that drop out from one school cannot transfer to the other school.
} 
that, for a given student, these are probabilities of completion for specified productivity and school type.

From the above it follows that

$$
p_{H 1}>p_{L 1}, p_{H 2}>p_{L 2}, p_{H 1}<p_{H 2} \text { and } p_{L 1}<p_{L 2}
$$

In our model, the unit of time is the standard duration of university studies (say, 4 years). At the end of period $t$ students from generation $t$ either graduate or drop out of the program. At the same time, students of generation $t+1$ enroll. Thus, different values of time index $t$ correspond to different (non-overlapping) generations of students. Let $n_{H 1}(t)$ be the number of high productivity students enrolling in $U_{1}$ at time $t$. We define $n_{H_{2}}(t)$, $n_{L 1}(t)$ and $n_{L 2}(t)$ in a similar fashion. We assume that the total number of high and low productivity students do not change over time, i.e. that $n_{H}=n_{H 1}(t)+n_{H 2}(t)$ and $n_{L}=n_{L 1}(t)+n_{L 2}(t)$ are constant. The number of students in each school at time $t$ is given by $n_{1}(t)=n_{L 1}(t)+n_{H 1}(t)$, and $n_{2}(t)=n_{L 2}(t)+n_{H 2}(t)$, respectively. We are interested in how the distribution of high and low productivity students changes over time.

Probabilities $p_{H 1}, p_{L 1}, p_{H 2}$ and $p_{L 2}$, the total number of students of each type per generation, $n_{H}$ and $n_{L}$, and values of $S_{H}$ and $S_{L}$ are public knowledge. On the other hand, student type is private information (it becomes known only after hiring). ${ }^{11}$

Upon graduation, students enter the job market for new graduates. All competitive employers know about job applicants before hiring them is which university they graduated from. In particular, they not know their type. Thus, graduating students receive a salary that corresponds to the employer's expectation of their productivity.

An applicant who graduated from $U_{i}, i=1,2$ at period $t$ receives a competitive offer ${ }^{12}$ :

$$
S_{i}(t)=q_{H i}(t) S_{H}+\left(1-q_{H i}(t)\right) S_{L} .
$$

Here, $q_{H i}(t)$ denotes employers belief about the proportion of high productivity graduates of $U_{i}$ at time $t$.

On the other hand, a prospective student selects a university that offers her higher expected salary. ${ }^{13}$ She knows her type and, thus, probability with which she would

\footnotetext{
${ }^{11}$ Problem of employer learning of productivity of employees is considered, among others in seminal paper Jovanovic (1979).

${ }^{12}$ Since we are interested in the market for new graduates, we normalize salaries of university dropouts to zero.

${ }^{13}$ While the cost of study is implicit in the probability of graduation, our model is not equivalent to the standard formulation with explicit cost.
} 
complete either program. Thus, her choice is determined by her expectation of postgraduation salaries for her cohort.

To determine enrollment dynamics, we model behavior of employers, who set wages, and prospective students, who decide which school to enroll into. Behavior of these players depends on the way in which they form expectations. There are several approaches to modeling expectations of economic agents (see Evans and Honkapohja (2001) for an excellent overview). The simplest one is nä̈ve or static expectation, in which the latest realization of the random variable is taken as the expectation of its future value. The adaptive expectation approach takes into the account all previous values of the variable. Both of these approaches are backward looking. Finally, the rational expectation approach assumes that the agent is forward looking, i.e. she knows the behavior of other agents in the model and uses this information in forming her expectations. As a benchmark, we start with a model in which both employers and students are rational (Section 3). In Section 4 we assume that employers are forward looking while students use naïve expectations. Finally, in Section 5 employers use adaptive (backward-looking) expectations while students are still naïve.

\section{Further notation}

Our system is completely determined by the set of parameters $\Omega=\left\{S_{H}, S_{L}, p_{H 1}, p_{L 1}\right.$, $\left.p_{H 2}, p_{L 2}, n_{H}, n_{L}\right\}$, which are common knowledge, initial student enrollment and the type of expectations used by the parties involved (students and the employers). For future reference, it is convenient to introduce some additional notation. In particular, we introduce quotient of salaries offered at time t, $Q(t)=\frac{S_{2}(t)}{S_{1}(t)}$ and its boundary values:

$$
Q_{\min }=\frac{S_{L}}{S_{H}} \text { and } Q_{\max }=\frac{S_{H}}{S_{L}} .
$$

If all students enrolled into $U_{i}$, the expected productivity of graduates would be:

$$
\bar{S}_{i}=\frac{n_{H} p_{H i}}{n_{H} p_{H i}+n_{L} p_{L i}} S_{H}+\frac{n_{L} p_{L i}}{n_{H} p_{H i}+n_{L} p_{L i}} S_{L}
$$

We refer to these as the limiting salaries. The ratio of limiting salaries is also important for our considerations, and we denote it by: $\bar{Q}=\frac{\overline{S_{2}}}{\bar{S}_{1}}$. 
In our analysis the ratio of probabilities for completing the two schools, for high and low productivity students will, also, play an important role. We denote these ratios, respectively, by $R_{H}=\frac{p_{H 1}}{p_{H 2}}$ and $R_{L}=\frac{p_{L 1}}{p_{L 2}}$. By assumption, $R_{H}<1, R_{L}<1$.

Let us also introduce $D_{i}=\frac{p_{H i}}{p_{L i}}, i=1,2$. These quantities measure distinction (differentiation) levels for each of the two schools - the bigger the corresponding ratio is, the more likely are high productivity students to complete the school versus the low productivity students, ceteris paribus. This ratio depends on the quality standards that the school sets and serves to differentiate between the two types of students. An increase in the distinction level improves the signal given to employers about the quality of their students, because it increases the likelihood that students graduating from this school are of high productivity.

Finally, we define by $R=\frac{R_{L}}{R_{H}}=\frac{D_{2}}{D_{1}}$ the ratio of distinction levels. Equilibrium enrollment in all approaches will strongly depend upon which university has greater distinction level, i.e. on the level of $R$. Note that the condition $D_{1}<D_{2}$ is equivalent to $R>1$. The following important proposition holds: ${ }^{14}$

Proposition 2.1. Condition $R>1$ is equivalent to $\bar{Q}>1$.

This Proposition tells us that the university with a higher distinction level also has a higher limiting salary. If all students are to choose the same university, they would receive higher salary if they graduate from the school which better differentiates between the two types based on probability of completion.

\section{Rational (RR) expectation approach}

In this section we assume that all agents form rational expectations. Both students and competitive employers are forward looking and know how the other side makes their expectations. The rationality assumption means that students and employers correctly anticipate behavior of each other in equilibrium. We model behavior of agents as a onestep game, repeated over time by different cohorts of students.

Students choose school that would offer them higher expected salary (recall that each student knows her type, i.e. probability of completion for each school). If a student's estimates of expected salaries are the same for the two universities, she could choose either one. Thus, both pure and mix strategy outcomes are possible.

\footnotetext{
${ }^{14}$ All proofs are in the Appendix.
} 
There are four possible outcomes in pure strategies:

$C_{1}$ - all prospective students select $U_{1}$

$C_{2}-$ all select $U_{2}$

$C_{3}$ - high productivity select $U_{1}$, while low productivity select $U_{2}$

$C_{4}$ - high productivity select $U_{2}$, while low productivity select $U_{1}$.

In addition, there are 5 possible mixed strategy outcomes:

$M_{1}$ - high productivity select $U_{1}$, while low productivity split choice

$M_{2}$-high productivity select $U_{2}$, while low productivity split choice

$M_{3}$-low productivity select $U_{1}$, while high productivity split choice

$M_{4}$-low productivity select $U_{2}$, while high productivity split choice

$M_{5}$ - both types split choice

To determine possible equilibria, the standard signaling games approach of Cho and Kreps (1987) is employed. It can be shown that, for generation of students at time $t$, Perfect Bayesian Nash Equilibrium $(\mathrm{PBE})^{15}$ is reached such that:

(i) Students' strategies are optimal given the employers' strategies (salary offers)

(ii) Employers' beliefs $q_{H 1}(t)$ and $q_{H 2}(t)$ are derived from students' strategies using Bayes' rule where possible.

(iii) Salaries offered to graduates of each university constitute a Nash equilibrium of the simultaneous-move wage offer game, in which the probability that the graduate of school $i=1,2$ is of high type is given by $q_{H i}(t)$.

Proposition 3.1 specifies PBEs in this model. ${ }^{16}$

Proposition 3.1: Possible PBEs are listed in Table 1.

Table 1

\section{Payoffs}

\section{Equilibrium Conditions}

High productivity Low productivity

students

students

\begin{tabular}{|c|c|c|}
\hline Pure $C_{1}$ & $\frac{S_{L}}{\bar{S}_{1}}<R_{H}$ and $\frac{S_{L}}{\bar{S}_{1}}<R_{L}$ & $p_{H 1} \bar{S}_{1}$ \\
\hline
\end{tabular}

\footnotetext{
${ }^{15}$ See, e.g. Mas-Colell, Whinston and Green (1995), p. 452.

${ }^{16}$ Proposition 3.1 specifies PBEs in all generic cases. In a non-generic case, there are also PBEs of type $M_{5}$, when $Q_{\operatorname{mi~}}<R_{H}=R_{L}<Q_{\max }$. We will not consider this case, which arises on a subset of $\Omega$ of measure zero.
} 


\begin{tabular}{llll}
\hline Pure $\boldsymbol{C}_{2}$ & Always & $p_{H 2} \bar{S}_{2}$ & $p_{L 2} \bar{S}_{2}$
\end{tabular}
Pure $C_{3}$
$R_{L}<Q_{\text {m i }}<R_{H}$
$p_{H 1} S_{H}$
$p_{L 2} S_{L}$

Mixed $M_{1} \quad Q_{\text {min }}<R_{L}<\frac{S_{L}}{\bar{S}_{1}}$ and $R_{L}<R_{H} \quad p_{L 2} D_{1} S_{L} \quad p_{L 2} S_{L}$

$\operatorname{Mixed} M_{4} \quad Q_{\text {min }}<R_{H}<\frac{\bar{S}_{2}}{S_{H}}$ and $R_{L}<R_{H} \quad p_{H 1} S_{H} \quad p_{L 2} R_{H} S_{H}$

From Proposition 3.1 we see that PBEs are not necessarily unique. Namely, we see that $C_{2}$ can always occur, and thus conditions for existence of every other equilibrium overlaps with that of $C_{2}$. In order to obtain unique equilibria for a given set of parameter values, we apply criterion of elimination of dominated equilibria and D1 criterion by Cho and Kreps (1987) ${ }^{17}$. The remaining unique equilibria with conditions under which they arise are stated in the Theorem 3.1.

Theorem 3.1: After applying elimination of dominated equilibria and D1 criterion, unique remaining equilibria are specified in Table 2

Table 2

Conditions for unique remaining PBEs

\begin{tabular}{cc}
\hline Equilibrium & Conditions \\
\hline Case $\boldsymbol{R}>\mathbf{1}$ & always \\
\hline Pure $\boldsymbol{C}_{\mathbf{2}}$ & Case $\boldsymbol{R}<\mathbf{1}$ \\
\hline Pure $\boldsymbol{C}_{\mathbf{1}}$ & $\frac{S_{L}}{\bar{S}_{1}}<R_{L}$ and $\frac{\bar{S}_{2}}{S_{H}}<R_{H}$ \\
\hline Pure $\boldsymbol{C}_{\mathbf{2}}$ & $R_{H}<\frac{\bar{S}_{2}}{S_{H}}$ \\
\hline
\end{tabular}

${ }^{17}$ We actually need the D1 condition for uniqueness. If we apply only dominance, intuitive criterion and Banks and Sobel divinity, we might not get uniqueness. For instance, when $\frac{\bar{S}_{2}}{S_{H}}<R_{L}<R_{H}<\bar{Q}$ and $\frac{S_{L}}{\bar{S}_{1}}<R_{L}$, both $C_{1}$ and $C_{2}$ would remain. 


\begin{tabular}{cc}
\hline Pure $\boldsymbol{C}_{3}$ & $R_{L}<Q_{\min }$ and $\frac{\bar{S}_{2}}{S_{H}}<R_{H}$ \\
\hline Mixed $M_{1}$ & $Q_{\min }<R_{L}<\frac{S_{L}}{\bar{S}_{1}}$ and $\frac{\bar{S}_{2}}{S_{H}}<R_{H}$ \\
\hline
\end{tabular}

These equilibria are reached in one generation (immediately) in RR approach. A graphical representation that illustrates the results of Table 2 can be found on Fig. 1. There, we fix probabilities of completion for the second school and present possible equilibrium outcomes for different values of probabilities of completion for more challenging school.

From Table 2 we see that when $R>1$, i.e. when easier to complete school differentiates better between high and low productivity students, all students will attend it if both employers and students are rational, i.e. $C_{2}$ obtains. The resulting equilibrium is pooling, i.e. employers cannot determine the quality of students based on the school they completed. In this case no student would ever attend more challenging university. On Fig. 1 this corresponds to the area above the main diagonal.

On the other hand, when the more challenging (first) university better differentiates between high and low productivity students, the situation becomes more complicated. Indeed, let us fix values of $R_{L}, R_{H}$ such that $R_{H}>R_{L}$. In this case, when the limiting salary for the second university is sufficiently large, all students will again choose it, i.e. equilibrium outcome would be $C_{2}$. However, when the limiting salary for the second university is below the threshold value, all high productivity students will enroll into the more challenging school. Behavior of the low productivity students will in this case depend on the value of the limiting salary for the first university - when it is sufficiently large, all low productivity students will prefer the first university. This corresponds to outcome $C_{1}$. Otherwise, low productivity students either split their choice between the two universities (mixed equilibrium outcome $M_{1}$ ), or all select the second university, if it offers better expected salaries because of lower dropout rates regardless of the salaries offered on the market. In the latter case, separating equilibrium $C_{3}$ is obtained. Only in that case, employer can uniquely determine types of students based on the school they complete.

The case when $R<1$ is presented in Fig. 1 below the main diagonal.

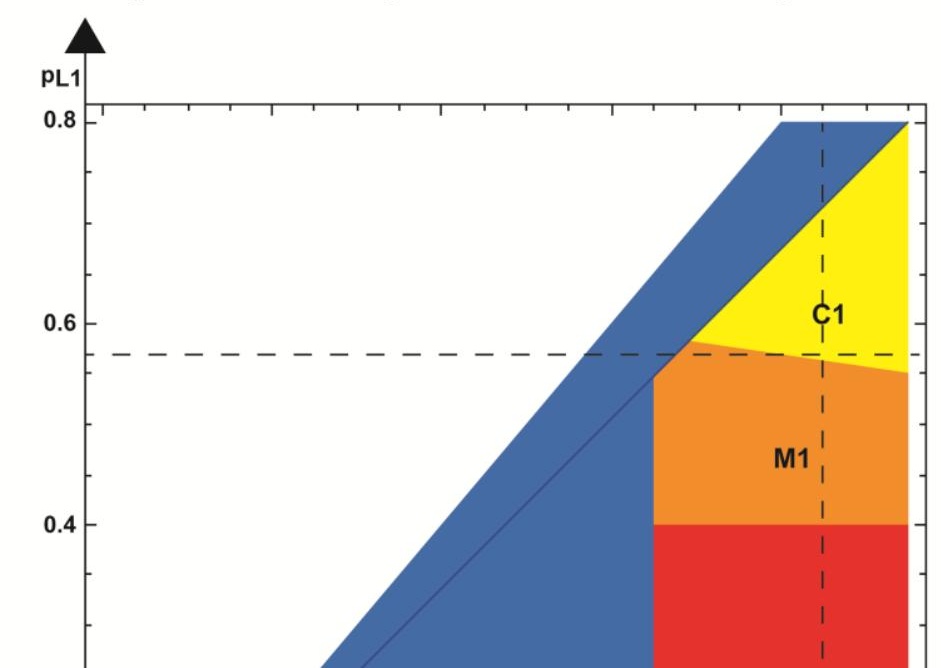


Fig. 1 
One can see that if probability of completion of the more challenging school by the high productivity type is small enough (for fixed values of the probabilities of completion at the less challenging school), all students select second school ( $C_{2}$ obtains). However, when this probability is above a threshold, other equilibria are possible and depend, also, on the probability of completion of the more challenging school by low productivity type. Namely, as one increases this probability, ceteris paribus, we obtain, respectively, $C_{3}, M_{1}$ and, finally, $C_{1}$. Thus, as it becomes easier for low productivity students to complete the more challenging school, it becomes more attractive to them.

The following Theorem discusses one important aspect of the comparative statics in RR approach.

Theorem 3.2: In RR approach, increasing probability of completion for high productivity students in either school, ceteris paribus, will never lead to a decrease of an equilibrium number of enrolled students of either type.

The intuition is simple. By increasing probability of completion of high productivity students, ceteris paribus, a university would raise their proportion in graduating class, thus increasing the expected productivity of school graduates. This makes the school more attractive to students of both types. This can be seen by following the horizontal dashed line in the direction of increasing values of $p_{H 1}$ on Fig. 1. While natural, this intuition may break down when full rationality assumption is relaxed. On the other hand, increasing $p_{L 1}$, ceteris paribus, i.e. moving upward along the vertical dashed line may initially attract higher number of low productivity students but, eventually, leads to the loss of all students at the first university.

\section{Rational expectations of the employer and naïve student expectations (RN approach)}

In this section we start relaxing rationality assumptions. In particular, from this section on we assume that, instead of using rational expectation when setting expectations regarding future salaries, students use naïve (or static) expectation approach. ${ }^{18}$ That means that, when making enrollment decisions, students use the latest salaries offered to graduates of the two schools as their expectation of prospective post-graduation wages. On the other hand, in this section we continue to assume that employers set their expectations rationally, i.e. based on their knowledge of the student behavior. In particular, this means that the employers know that students will make their choice of university using naïve expectations about prospective salaries. As a result, employers are able to correctly predict what choice students of each type will make.

${ }^{18}$ For general discussion on expectation formation see, e.g. Evans and Honkapohja (2001). Student expectations are addressed, among others, in Manski (1989, 1993), Wiswall and Zafar (2011) and Zafar (2011). 
The approach in this section is analogous to the dynamic market model of Noldeke and Samuelson (1997) in which buyers (employers in our model) buy high or low quality goods from sellers (hire students) based on a signal sent by sellers (school selection by students). Aside from the fact that we use probabilities of graduation instead of fixed cost of studies (as is customarily done in Spence-like models), there is another difference with their model. Namely, in our model types of expectations of agents are reversed with respect to theirs: while buyers there use naïve expectations and sellers use rational ones, in our model employers are rational and students are naïve.

The salary offered to the initial generation is not determined by our rationality assumption. We consider two ways in which initial salary in RN case can be set.

Natural initial salaries assumption (RNN): A natural assumption about the initial enrollment that employers can make is that the proportion of students of the two types enrolling at each university is the same as their proportion in the entire population. In this case, the initial employers' beliefs of proportion of high productivity graduates is:

$$
q_{H i}(1)=\frac{n_{H} p_{H i}}{n_{H} p_{H i}+n_{L} p_{L i}}, i=1,2
$$

so the initial salaries are set as $S_{i}(1)=\bar{S}_{i}$ (see Equation (4)).

General initial salaries approach (RNG): The other approach is to take employers' estimates of initial enrollment, $\hat{n}_{H i}(0)$ and $\hat{n}_{L i}(0)$, as free parameters subject only to the constraints that $\hat{n}_{H 1}(0)+\hat{n}_{H 2}(0)=n_{H}$ and $\hat{n}_{L 1}(0)+\hat{n}_{L 2}(0)=n_{L}$.

In that case initial employers' beliefs are given by:

$$
q_{H i}(1)=\frac{\hat{n}_{H i}(0) p_{H i}}{\hat{n}_{H i}(0) p_{H i}+\hat{n}_{L i}(0) p_{L i}}, i=1,2,
$$

and initial salaries (salaries at time 1) are set according to Equation (2). The following proposition states conditions that the initial salaries need to satisfy. ${ }^{19}$

Proposition 4.1. One of the following two cases has to hold:

(i) $\quad \bar{S}_{1} \leq S_{1}(1)$ and $\bar{S}_{2} \geq S_{2}(1)$

(ii) $\quad \bar{S}_{1} \geq S_{1}(1)$ and $\bar{S}_{2} \leq S_{2}(1)$.

In addition, $S_{L} \leq S_{i}(1) \leq S_{H}$.

\footnotetext{
${ }^{19}$ The proof is a special case of the proof of Proposition 5.1 in the Appendix.
} 
Note that for arbitrary $S_{i}(1)$ satisfying conditions stated in Proposition 4.1 there exist employers' estimates of initial enrollment $\left(\hat{n}_{H i}(0), \hat{n}_{L i}(0)\right)$ that give rise to these salary offers.

In RNG approach, enrollment dynamics depends on the initial employers' estimates only through the initial salaries. For this reason, in what follows when we talk about the initial conditions we mean the initial salaries.

\section{Student choices after initial enrollment}

What happens at a given subsequent time period $t$ ? Students make their decisions based on what they believe about prospective salaries upon graduation (in this and the next section they use naïve expectations) as well as probabilities of completion. Suppose, e.g., that both types have higher expectation of salary if they enroll into the more challenging school, i.e. that: ${ }^{20}$

$$
p_{H 1} S_{1}(t)>p_{H 2} S_{2}(t), p_{L 1} S_{1}(t)>p_{L 2} S_{2}(t)
$$

In that case, which we, as in Section 3, denote with $C_{1}$, both types select $U_{1}$ at time $t .^{21}$ More generally, consider a one-step game at time $t$. There are four possible outcomes, two pooling one-step outcomes, $C_{1}$ and $C_{2}$, and two separating ones, $C_{3}$ and $C_{4}$. We will refer to these one-step outcomes as "states" when we later study dynamics of the model.

Conditions (7) are equivalent to: $Q(t)<R_{H}$ and $Q(t)<R_{L}$. When the ratio of salaries is $Q(t)$ is lower than $R_{H}$, high productivity students select the more challenging university. Similarly, low productivity students select the more challenging university if the ratio of salaries is lower than $R_{L}$. Thus, $R_{H}$ and $R_{L}$ are thresholds values that determine what schools students select at a given point in time. While $Q(t)$ changes over time, the thresholds do not change over time, and depend only on completion rates for the two Universities, which are exogenously given. ${ }^{22}$

We summarize the above considerations in Table 3.

Table 3

\footnotetext{
${ }^{20}$ If no students enroll at a given university at time $t$, the last salary offer for that university graduates is used by students as the estimate of their prospective post-graduation salary. Similar assumption is made in Noldeke and Samuelson (1997). Together with the initial salaries choice (RNN or RNG approaches), this defines salaries at every point in time.

${ }^{21}$ At each point in time after the initial generation all students of the same type make identical school selection.

${ }^{22}$ Note that in this paper we do not consider cases when $Q(t)$ is equal to a threshold value, which happens on a non-generic set of measure zero of parameters and initial conditions, i.e. almost never.
} 
Possible student choices

\begin{tabular}{lcccc}
\hline Student choice & $C_{1}$ & $C_{2}$ & $C_{3}$ & $C_{4}$ \\
\hline \multirow{3}{*}{ Conditions } & $Q(t)<R_{H}$ & $Q(t)>R_{H}$ & $Q(t)<R_{H}$ & $Q(t)>R_{H}$ \\
& $Q(t)<R_{L}$ & $Q(t)>R_{L}$ & $Q(t)>R_{L}$ & $Q(t)<R_{L}$ \\
\hline
\end{tabular}

\section{Enrollment dynamics in RN approach}

In the case of RNN dynamics is specified by Theorem 4.1 .

Theorem 4.1. In RNN approach, when $R>1$ the system is in state $C_{2}$ at all times while when $R<1$, possible dynamics with conditions under which they occur are given in Table 4.

Table 4

State sequences for $R<1$ in RNN approach

\begin{tabular}{|c|c|c|c|c|c|c|c|c|}
\hline \multirow{3}{*}{$\begin{array}{l}\text { Long run } \\
\text { equilibria }\end{array}$} & \multirow{3}{*}{ Case } & \multicolumn{6}{|c|}{ Dynamics } & \multirow{3}{*}{ Conditions } \\
\hline & & \multicolumn{2}{|c|}{$t=1$} & \multicolumn{2}{|c|}{$t=2$} & \multicolumn{2}{|c|}{$t=3$} & \\
\hline & & State & $Q$ & State & $Q$ & State & $Q$ & \\
\hline \multirow[b]{2}{*}{$C_{1}$} & 1 & $C_{1}$ & $\bar{Q}$ & $C_{1}$ & $\bar{Q}$ & $C_{1}$ & $\bar{Q}$ & $\bar{Q}<R_{L}$ \\
\hline & 2 & $C_{3}$ & $\frac{S_{L}}{S_{H}}$ & $C_{1}$ & $\frac{S_{L}}{\bar{S}_{1}}$ & $C_{1}$ & $\frac{S_{L}}{\overline{S_{1}}}$ & $R_{L}<\bar{Q}<R_{H}, \frac{S_{L}}{\bar{S}_{1}}<R_{L}$ \\
\hline$C_{2}$ & 3 & $C_{2}$ & $\bar{Q}$ & $C_{2}$ & $\bar{Q}$ & $C_{2}$ & $\bar{Q}$ & $\bar{Q}>R_{H}$ \\
\hline$C_{3}$ & 4 & $C_{3}$ & $\frac{S_{L}}{S_{H}}$ & $C_{3}$ & $\frac{S_{L}}{S_{H}}$ & $C_{3}$ & $\frac{S_{L}}{S_{H}}$ & $\bar{Q}<R_{H}, Q_{\mathrm{min}}>R_{L}$ \\
\hline $\begin{array}{c}C_{1}-C_{3} \\
\text { cycle }\end{array}$ & 5 & $C_{3}$ & $\frac{S_{L}}{S_{H}}$ & $C_{1}$ & $\frac{S_{L}}{\bar{S}_{1}}$ & $C_{3}$ & $\frac{S_{L}}{S_{H}}$ & $\bar{Q}<R_{H}, Q_{\min }<R_{L}, R_{L}<\frac{S_{L}}{\bar{S}_{1}}$ \\
\hline
\end{tabular}

Here, $C_{1}-C_{3}$ denotes infinitely repeated cycle, alternating after every time step between the two states. For the other states, the right-most state represents the steady state (longrun equilibrium).

On the other hand, in RNG approach dynamics is described by Theorem 4.2.

Theorem 4.2.Possible state dynamics in RNG approach, together with conditions when they occur, are given in Table A. 1 for $R>1$ and A. 2 for $R<1$ (see Appendix).

\section{Summary and discussion of the results in RN approach}

Using Theorems 4.1 and 4.2, we can characterize all possible long run equilibria in RN approach. 
Theorem 4.3. After at most 2 generations (by the time $t=2$ ) in RNN approach and at most 3 generations (by the time $t=3$ ) in RNG approach, one of the following long run equilibria will occur:

(i) All students enroll in the more challenging university $\left(C_{1}\right)$

(ii) All students enroll in the less challenging university $\left(C_{2}\right)$

(iii) High productivity students enroll in the more challenging university while low productivity ones enroll in the less challenging university $\left(C_{3}\right)$

(iv) High productivity students enroll in the more challenging university while low productivity students alternate enrollment in two universities after every time step, indefinitely (cycle $\left.C_{1}-C_{3}\right)^{23}$

The conditions which determine which of the long run equilibria occurs are given in the Tables 5 and 6.

Table 5

Conditions for long run equilibria for $R<1$

\begin{tabular}{|c|c|c|}
\hline Equilibrium & General conditions & Initial conditions \\
\hline Pure $C_{1}$ & $\frac{S_{L}}{\bar{S}_{1}}<R_{L}$ & $\begin{array}{c}Q(1)<R_{L}, . \frac{S_{2}(1)}{\bar{S}_{1}}<R_{H} \text { or } \\
Q(1)>R_{H}, \frac{\bar{S}_{2}}{S_{1}(1)}<R_{H}\end{array}$ \\
\hline Pure $C_{2}$ & Always & $\begin{array}{c}Q(1)>R_{H}, \frac{\bar{S}_{2}}{S_{1}(1)}>R_{H} \text { or } \\
Q(1)<R_{L}, \frac{S_{2}(1)}{\bar{S}_{1}}>R_{H} \text { or } \\
R_{L}<Q(1)<R_{H}, Q_{\min }<R_{L}, \frac{S_{L}}{\bar{S}_{1}}>R_{H}\end{array}$ \\
\hline Pure $C_{3}$ & $R_{L}<Q_{\mathrm{min}}<R_{H}$ & $\begin{array}{c}\frac{\bar{S}_{2}}{S_{1}(1)}<R_{H} \text { or } \\
Q(1)<R_{H}\end{array}$ \\
\hline Cycle $C_{1}-C_{3}$ & $Q_{\min }<R_{L}<\frac{S_{L}}{\bar{S}_{1}}$ & $\begin{array}{c}Q(1)<R_{L}, \frac{S_{2}(1)}{\bar{S}_{1}}<R_{H} \text { or } \\
R_{L}<Q(1)<R_{H}, \frac{S_{L}}{\bar{S}_{1}}<R_{H} \text { or } \\
Q(1)>R_{H}, \frac{\bar{S}_{2}}{S_{1}(1)}<R_{H}\end{array}$ \\
\hline
\end{tabular}

${ }^{23}$ The cycles in signaling models were first considered in Spence (1974). Noldeke and Samuelson (1997) characterized cycle equilibria in signaling models and showed that cycles consist of two states. 
Table 6

Conditions for long run equilibria for $R>1$

\section{Equilibrium $\quad$ General conditions $\quad$ Initial conditions}

\begin{tabular}{ccc}
\hline Pure $C_{1}$ & $\frac{S_{L}}{\bar{S}_{1}}<R_{H}$ & $\frac{S_{2}(1)}{\bar{S}_{1}}<R_{H}$ \\
\hline Pure $C_{2}$ & Always & $\frac{S_{2}(1)}{\bar{S}_{1}}>R_{H}$ \\
\hline
\end{tabular}

Theorems 4.1-3 show that there are four possible long run equilibrium outcomes under the assumptions made in this section. The first two, $C_{1}$ and $C_{2}$, are pooling equilibria in which one of the universities eventually runs out of students. The third, $C_{3}$, is perfectly separating one in which low productivity students ultimately always choose university that is easier to complete, while high productivity ones select the more challenging school. Finally, there is a possibility of a cyclic student enrollment, $C_{1}-C_{3}$. In that case the less challenging school is in every other generation left without students, after which low productivity ones return to it. ${ }^{24}$

Let us compare more closely $\mathrm{RN}$ and the benchmark RR approach. There are two types of conditions that determine the long run equilibrium outcomes in $\mathrm{RN}$ approach (see Tables 5 and 6). These can be split into general and initial conditions. The general conditions are the same as those in case of rational expectations prior to eliminating multiple Nash equilibria (see Table 1), with a cycle in $\mathrm{RN}$ case corresponding to the mixed equilibrium $M_{1}$ in RR case. Moreover, every sequential Nash equilibrium from Table 1, except for $M_{4}$, has corresponding long run equilibrium in RN approach and can be realized under suitable initial conditions. The $M_{4}$ equilibrium in RR would correspond to a cycle of alternating states $C_{2}$ and $C_{3}$, which is not possible in RN dynamics. Note, however, that $M_{4}$ does not survive the elimination procedure (see Section 3 ).

One novelty in a mixed rational-naïve (RN) approach compared to the fully rational (RR) approach is that, in contrast to $\mathrm{RR}$, in $\mathrm{RN}$ case it may take more than one generation of students to reach a long run equilibrium outcome. In addition, in RN case these long run outcomes, generally, depend on the initial conditions. This dependence is important only for RNG case. In the case of RNN, the initial salaries are not a free parameter and it makes sense to compare conditions determining long run equilibria (Table 4) to the analogous conditions for RR case after the process of multiple equilibrium elimination is completed (Table 2). The conditions are very similar, the only difference being that when

\footnotetext{
${ }^{24} \mathrm{We}$ assume that as long as there is a chance for a university to recover its attractiveness, it would remain open.
} 
$R<1$ the expression $\frac{\bar{S}_{2}}{S_{H}}$ in RR approach needs to be replaced with $\bar{Q}$ in RNN approach. The effect of this is that $C_{2}$ region from Fig. 1 is now enlarged, ceteris paribus: vertical line separating the region $C_{2}$ from the other regions on the right is replaced by a hyperbola (see Fig. 2).

Comparative statics becomes more interesting in case of the RNG and AN approaches, namely analogue of the Theorem 3.2 does not hold in these cases generically. This is illustrated in Fig. 3 (see, also, Section 6).

Following the horizontal dashed line in the direction of increasing values of $p_{H 1}$ on Fig. 3 , region $C_{2}$ is followed by $C_{1}-C_{3}$. Further increase in $p_{H 1}$ moves us back to $C_{2}$ and then, again, to $C_{1}-C_{3}$. Finally, when $p_{H 1}$ is large enough we end up in $C_{1}$. In other words, increasing the probability of completion of high productivity students at the first school has a non-monotonic effect on the number of students entering that university, ceteris paribus, in contrast to RR and RNN cases.

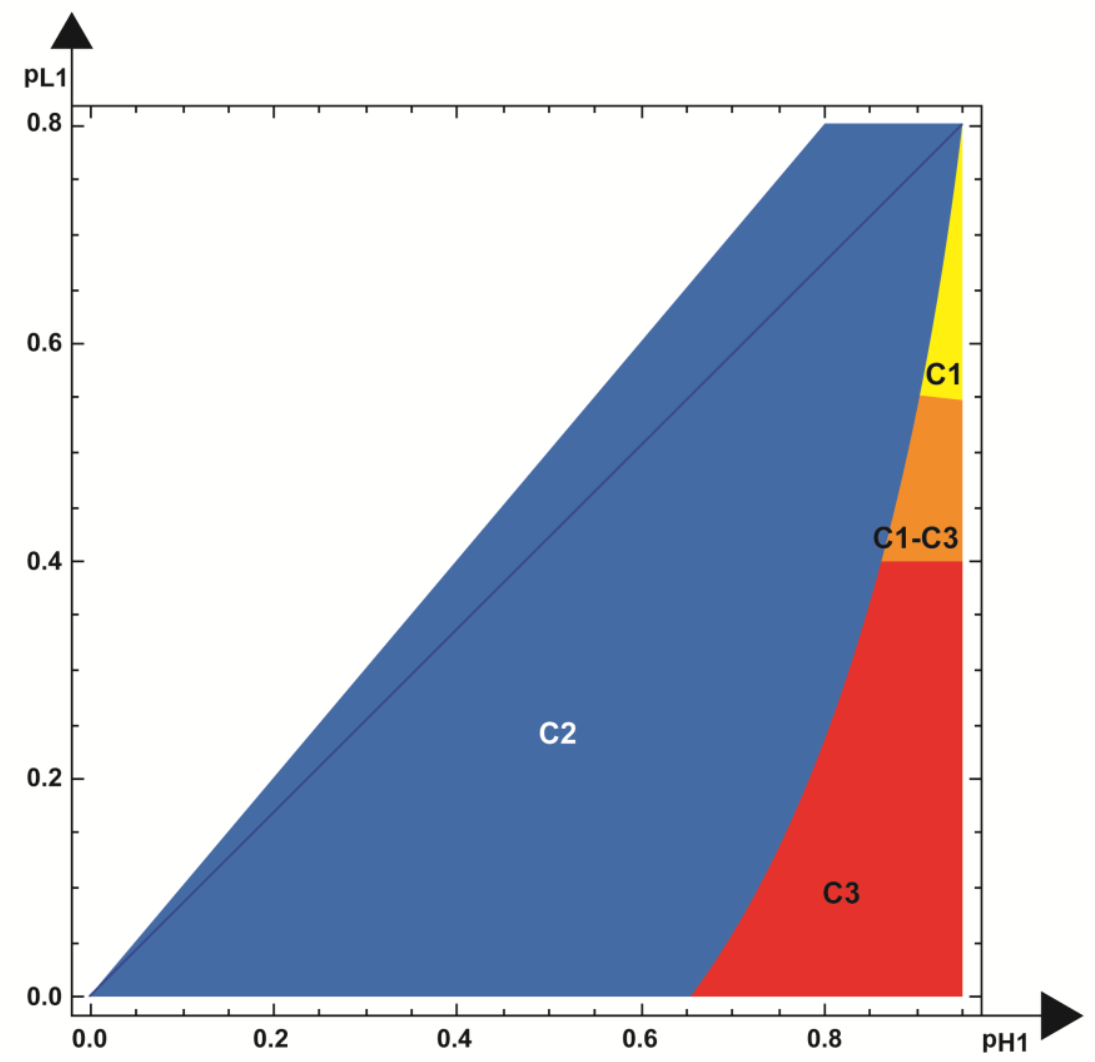

Fig. 2 


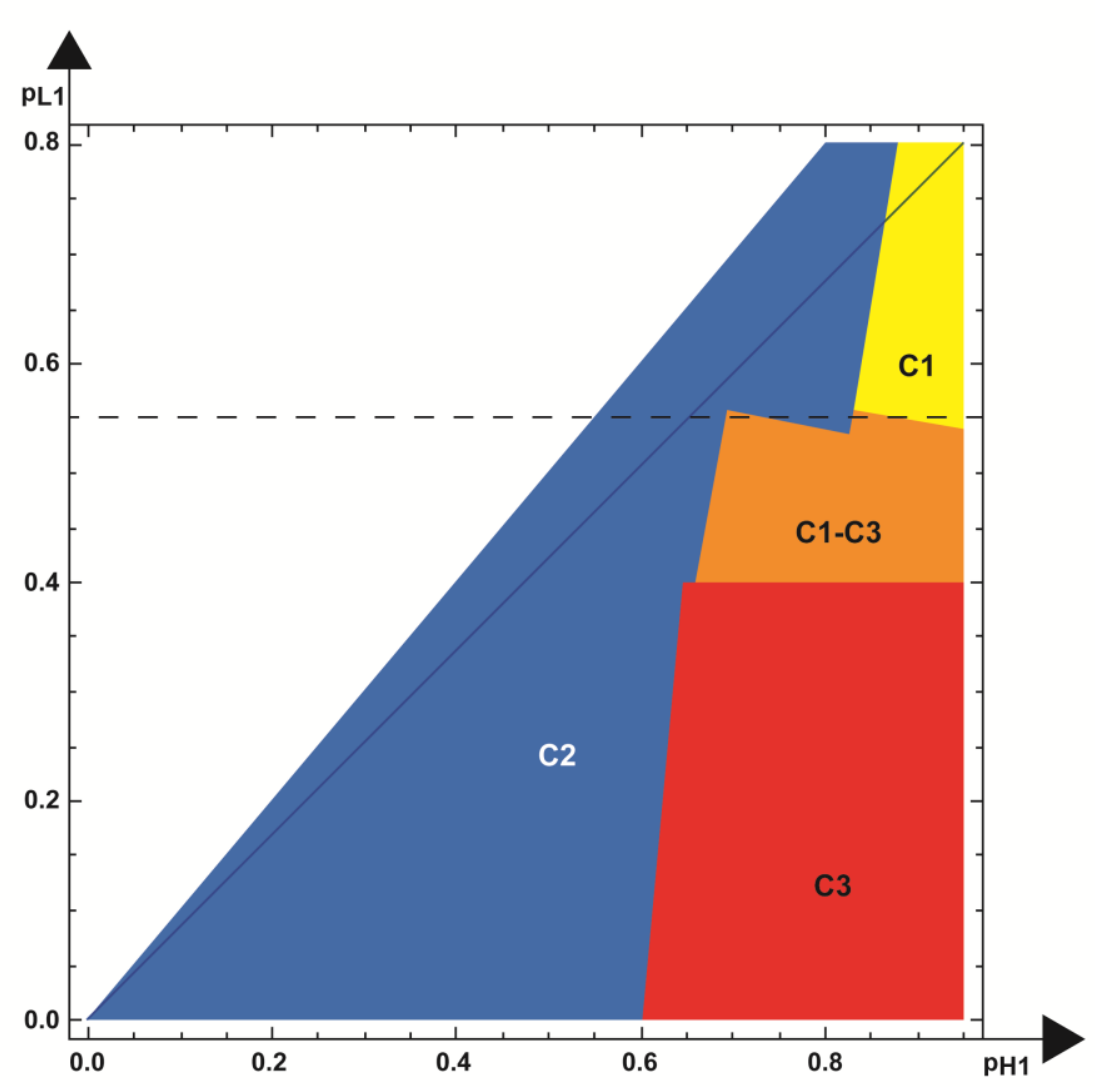

Fig. 3

\section{Adaptive expectations of the employer and naïve expectations of students (AN approach)}

In this section, we continue to reduce the assumptions of rationality allowing now employers to use adaptive (backwards looking) instead of rational (forward looking) expectations. This assumption arises naturally when one wants to take into consideration reputation of schools. Namely, we assume that in forming their salary offers to graduates employers take into the account productivity of all students that have been employed up to that point, adaptively adjusting their beliefs about the number of students of a given type graduating from each school. Employers keep on record, for every worker, information about which school she graduated from and what is her productivity level. ${ }^{25}$ Thus, the salary offer depends on the reputation earned by each school up to that point. We assume that prospective students of the next generation make enrollment decisions

\footnotetext{
${ }^{25} \mathrm{We}$ assume that all graduates get employed after graduation and that the productivity level of each is revealed to the employer immediately upon hiring. Employers update their beliefs with each additional graduate that gets hired.
} 
after all graduates of the previous generation get employed. Like in Section 4, students use naïve expectations in making enrollment decisions taking salary offer at the end of the employment period as their estimate of post-graduation salary.

\section{Adjusting employer beliefs and corresponding salaries}

First, let us introduce some notation. The total number of high productivity students that enrolled into $U_{i}$ up to time $t$ is:

$$
N_{H i}(t)=\sum_{\tau=0}^{t} n_{H i}(\tau)
$$

We define $N_{L i}(t)$ analogously. Given the assumptions of Section 2, the number of high and low productivity students graduating from $U_{i}$ up to generation $t$ is $N_{H i}(t-1) \cdot p_{H i}$ and $N_{L i}(t-1) \cdot p_{L i}$, respectively.

The salary offer to graduates of $U_{i}$ at the time of enrollment decisions of generation $t$ is given by expression (2) with:

$$
q_{H i}(t)=\frac{N_{H i}(t-1) p_{H i}}{N_{H i}(t-1) p_{H i}+N_{L i}(t-1) p_{L i}}
$$

Note that in adaptive employers' expectations, information on the graduating class from each year carries equal weight. ${ }^{26}$

Proposition 5.1 establishes important relationships between limiting and current salaries.

Proposition 5.1. At any given time $t$, in AN approach, one of the following two cases holds:

(i) $\quad \bar{S}_{1} \leq S_{1}(t)$ and $\bar{S}_{2} \geq S_{2}(t)$

(ii) $\quad \bar{S}_{1} \geq S_{1}(t)$ and $\bar{S}_{2} \leq S_{2}(t)$.

\section{Dynamics in AN approach}

Like in Section 4, student choices at time $\mathrm{t}$ are driven by quotient of salaries $Q(t)$. Evolution in one time step is described by Theorem 5.1.

\footnotetext{
${ }^{26}$ In unreported robustness check, we consider more general adaptive expectations in which weight of each passing generation declines exponentially, i.e. older information about students is deemed less informative than new information (see, also, Evans and Honkapohja (2001)) and demonstrate that qualitative features of the model remain intact.
} 
Theorem 5.1.In AN approach, the following holds:

(i) If the system at time $t$ is in states $C_{1}$ or $C_{2}$ then $Q(t+1)$ is between $Q(t)$ and $\bar{Q}$ , i.e. $Q$ moves towards $\bar{Q}$ monotonically.

(ii) If the system at time $t$ is in state $C_{3}$ then $Q(t+1)$ is between $Q(t)$ and $Q_{\min }$, i.e. $Q$ decreases towards $Q_{\min }$.

(iii) If the system at time $t$ is in state $C_{4}$ then $Q(t+1)$ is between $Q(t)$ and $Q_{\max }$ i.e. $Q$ increases towards $Q_{\max }$.

Dynamics is described by Theorem 5.2.

Theorem 5.2. All possible state dynamics, together with conditions when they occur, in AN approach correspond to cases in Table A.4 for $R>1$ and Table A.5 for $R<1$.

Using Theorem 5.2, we can characterize all possible long run equilibria.

Theorem 5.3. After a sufficient number of generations, the following long run equilibria can occur in AN approach:

(i) All students enroll in the more challenging university $\left(C_{1}\right)$

(ii) All students enroll in the less challenging university $\left(C_{2}\right)$

(iii) High productivity students enroll in the more challenging university while low productivity students enroll in the less challenging university $\left(C_{3}\right)$

(iv) High productivity students enroll in the more challenging university while low productivity students alternate enrollment in two universities indefinitely (cycle $C_{1}-C_{3}$ ). Each state in the cycle can be repeated several times before the system moves to the other state.

General conditions that determine which of the long run equilibria can occur are given in tables 4 and 5, i.e. they are the same as in the RN model. On the other hand, initial condition dependence of long term equilibria is more complex, and is given in Tables A.4 and A.5.

The main difference between $\mathrm{RN}$ and $\mathrm{AN}$ equilibrium is that in case of AN approach, as we shall see in the examples below, it may take a considerably longer time than in RN case to reach long term equilibrium outcome. Namely, in contrast to RN approach, in case of AN some of the states may be repeated for several generations before a move to another state occurs. The reason for this is that, when employers are backward-looking, offered salaries may change even if the state does not. When either of the long-run equilibria $C_{1}, C_{2}$ or $C_{3}$ is realized, salary offers in AN approach gradually converge to the same levels that are reached immediately in corresponding RN and RR cases. Moreover, in cycle $C_{1}-C_{3}$ salary offers converge to the same levels as in mixed equilibrium $M_{1}$ in RR approach. 


\section{Applications}

We now examine behavior of our model in some characteristic situations. In the examples that we consider, productivity levels are set as 1 and 2, (for the low and high productivity students, respectively). Furthermore, we assume that, per annum, there are 1000 low productivity and 500 high productivity students. For each example, we compare dynamics in four (RR, RNN, RNG, and AN) approaches.

Example 6.1 Let us first consider a situation in which more challenging school has high distinction level between high and low productivity students, while the less challenging university has a much lower distinction level and is, at the same time, much easier to complete for the low productivity students. For the parameter values in Table 7, a separating equilibrium $\left(\boldsymbol{C}_{\mathbf{3}}\right)$ is obtained in the long run irrespectively of the way in which students and employers form expectations. In that equilibrium high productivity students attend more challenging university, while low productivity students opt for the less challenging school.

Table 7

Parameter values for Example 6.1

\begin{tabular}{|c|c|c|c|c|c|c|c|c|c|c|c|}
\hline & \multicolumn{2}{|c|}{$\begin{array}{c}\text { Initial } \\
\text { enrolment }\end{array}$} & \multicolumn{2}{|c|}{ Probabilities } & \multicolumn{2}{|c|}{$\begin{array}{c}\text { Initial } \\
\text { salaries }\end{array}$} & \multirow[t]{2}{*}{$\overline{S_{1}}$} & \multirow[t]{2}{*}{$\bar{S}_{2}$} & \multirow{2}{*}{$\begin{array}{l}\text { Quotient of } \\
\text { probabilities } \\
\left(R_{\mathrm{L}} \text { and } R_{\mathrm{H}}\right)\end{array}$} & \multicolumn{2}{|c|}{$\begin{array}{c}\text { Distinction } \\
\text { levels }\end{array}$} \\
\hline & $n_{1}$ & $n_{2}$ & $p_{1}$ & $p_{2}$ & $S_{1}(0)$ & $S_{2}(0)$ & & & & $D_{1}$ & $D_{2}$ \\
\hline Low & 500 & 500 & 0.20 & 0.85 & \multirow{2}{*}{1.62} & \multirow{2}{*}{1.40} & \multirow{2}{*}{1.67} & \multirow{2}{*}{1.36} & 0.235 & \multirow{2}{*}{4.00} & \multirow{2}{*}{1.12} \\
\hline High & 200 & 300 & 0.80 & 0.95 & & & & & 0.842 & & \\
\hline
\end{tabular}

Note that while the first university makes better distinction between the two groups of students than the second one, initially the enrolment of high productivity students is slightly skewed in favor of the second university.

\section{RR approach}

When both students and employers are fully rational (forward-looking), initial enrollment does not influence the equilibrium outcome. From Theorem 3.1, since our parameters satisfy conditions $R_{L}<Q_{\mathrm{min}}$ and $\frac{\bar{S}_{2}}{S_{H}}<R_{H}$, it follows that equilibrium state $C_{3}$ is reached in one step. In other words, high productivity students attend the first university, while low productivity students attend the second one.

\section{RNN approach}

In this case, in accordance with the results presented in Table 4, again state $C_{3}$ is reached in one step, since $R_{L}<Q_{\text {min }}$ and $\bar{Q}<R_{H}$. 


\section{RNG approach}

In this case initial enrollment plays an important role. With our choice of parameter and initial enrollment values a sequence of states corresponding to case 9 in Table A.2 (see the Appendix) is obtained. While in the first step all students enroll into the second university, in the second, final, step separating equilibrium $C_{3}$ is obtained as the long run outcome.

\section{AN approach}

In this case, the dynamics corresponds to case 11 in Table A.4 (see the Appendix). Like in the RNG case, the system is first in state $C_{2}$, eventually moving to state $C_{3}$. In contrast to the RNG case, state $C_{2}$ is repeated 13 times before the long run equilibrium state $C_{3}$ is reached. Thus, the main effect of reduction of employer rationality is an increase in the number of steps in which long run equilibrium state is obtained.

Fig. 4 illustrates dynamics in the AN approach. The quotient of salaries $(Q)$ determines which state the system is in at each point in time. When $Q$ is above $R_{H}$ (relative likelihood of completion of the first vs. the second school for high productivity students), the system is in state $C_{2}$, i.e. everybody choses the second school. When $Q$ is below the threshold $R_{H}$ (and above $R_{L}$, not presented on graph), the system is in state $C_{3}$, in which it stays indefinitely.

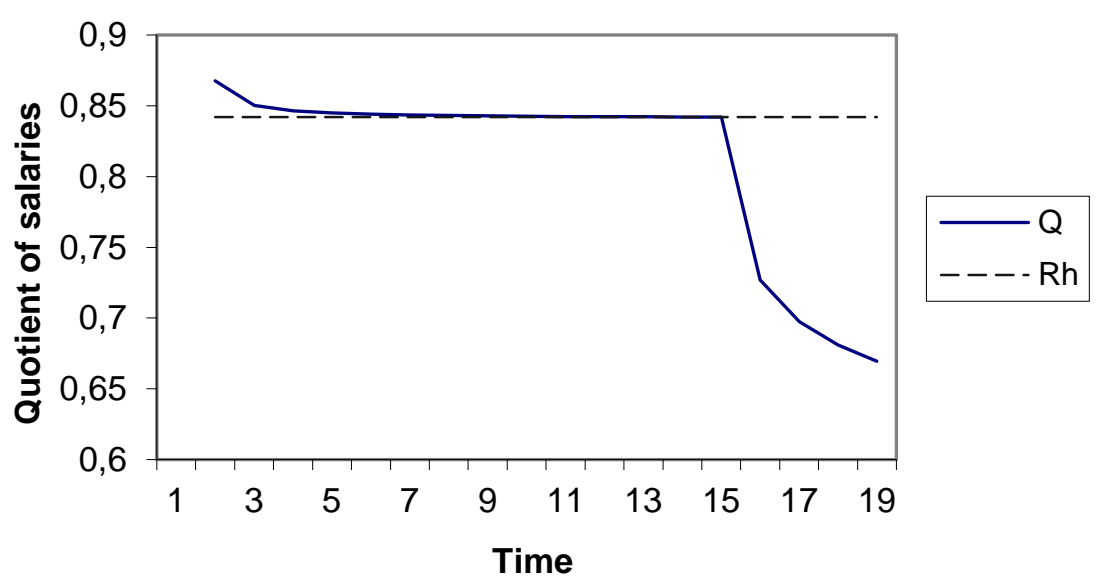

Fig. 4

$\mathrm{C}_{2}-\mathrm{C}_{3}$ dynamics in $\mathrm{AN}$ approach

Example 6.2 Now we consider the case when the university that is easier to complete also better differentiates between high and low productivity students $(R>1)$. In case of full rationality, condition $R>1$ ensures that all students select the second, easier to complete school. Interestingly, when the assumption of full rationality is relaxed, this outcome can be reversed. Namely, there exists a possibility that all students would, eventually, in that case attend the more challenging university. Thus, the way 
expectations are formed may in fact significantly impact the long run outcome. To illustrate this, we consider two sets of parameters (Tables 8 and 9) that differ from each other only in that the less challenging school in the second case makes life just a little bit harder for the higher productivity group, by reducing probability of their graduation from $90 \%$ to $80 \%$.

Table 8

Parameters for Example 6.2 (with higher $D_{2}$ )

\begin{tabular}{|c|c|c|c|c|c|c|c|c|c|c|c|}
\hline & \multicolumn{2}{|c|}{$\begin{array}{c}\text { Initial } \\
\text { enrolment }\end{array}$} & \multicolumn{2}{|c|}{ Probabilities } & \multicolumn{2}{|c|}{$\begin{array}{c}\text { Initial } \\
\text { salaries }\end{array}$} & \multirow[t]{2}{*}{$\overline{S_{1}}$} & \multirow{2}{*}{$\bar{S}_{2}$} & \multirow{2}{*}{$\begin{array}{c}\text { Quotient of } \\
\text { probabilities } \\
\left(R_{\mathrm{L}} \text { and } R_{\mathrm{H}}\right)\end{array}$} & \multicolumn{2}{|c|}{$\begin{array}{l}\text { Distinction } \\
\text { levels }\end{array}$} \\
\hline & $n_{1}$ & $n_{2}$ & $p_{1}$ & $p_{2}$ & $S_{1}(0)$ & $S_{2}(0)$ & & & & $D_{1}$ & $D_{2}$ \\
\hline Low & 200 & 800 & 0.10 & 0.15 & \multirow{2}{*}{1.93} & \multirow{2}{*}{1} & \multirow{2}{*}{1.71} & \multirow{2}{*}{1.75} & 0.667 & \multirow{2}{*}{5.00} & \multirow{2}{*}{6.00} \\
\hline High & 500 & 0 & 0.50 & 0.90 & & & & & 0.556 & & \\
\hline
\end{tabular}

Table 9

Parameters for Example 6.2 (with lower $D_{2}$ )

\begin{tabular}{|c|c|c|c|c|c|c|c|c|c|c|c|}
\hline & \multicolumn{2}{|c|}{$\begin{array}{c}\text { Initial } \\
\text { enrolment }\end{array}$} & \multicolumn{2}{|c|}{ Probabilities } & \multicolumn{2}{|c|}{$\begin{array}{c}\text { Initial } \\
\text { salaries }\end{array}$} & \multirow[t]{2}{*}{$\overline{S_{1}}$} & \multirow{2}{*}{$\bar{S}_{2}$} & \multirow{2}{*}{$\begin{array}{c}\text { Quotient of } \\
\text { probabilities } \\
\left(R_{\mathrm{L}} \text { and } R_{\mathrm{H}}\right)\end{array}$} & \multicolumn{2}{|c|}{$\begin{array}{l}\text { Distinction } \\
\text { levels }\end{array}$} \\
\hline & $n_{1}$ & $n_{2}$ & $p_{1}$ & $p_{2}$ & $S_{1}(0)$ & $S_{2}(0)$ & & & & $D_{1}$ & $D_{2}$ \\
\hline Low & 200 & 800 & 0.10 & 0.15 & \multirow{2}{*}{1.93} & \multirow[t]{2}{*}{1} & \multirow{2}{*}{1.71} & \multirow{2}{*}{1.73} & 0.667 & \multirow{2}{*}{5.00} & \multirow{2}{*}{5.33} \\
\hline High & 500 & 0 & 0.50 & 0.80 & & & & & 0.625 & & \\
\hline
\end{tabular}

\section{RR approach}

In this approach, initial enrollment does not influence the long-run outcome. This outcome is reached in one step. In both cases all students attend the less challenging university $\left(\boldsymbol{C}_{\mathbf{2}}\right)$, the only possibility in RR approach when $R>1$.

\section{RNN approach}

Again, all students enroll into the second university from the beginning, in both cases, in accordance with Theorem 4.1, since $R>1$.

\section{RNG approach}

In this approach, the two sets of parameters lead to diametrically opposite long run outcomes. In the first case (see Table 8) just like in RR and RNN approaches, in the long run all students attend the less challenging school. In contrast, in the second case (Table 9) all students attend the more challenging school at all times.

Even though the long run outcome in the first case is expected, the way in which it is realized is quite intriguing. Namely, in the first step after the initial enrollment all students opt for the more challenging school. Because the initial fraction of low productivity students at that school is smaller than in the general student population, this leads to a reduction in the expected productivity of its graduates. As a result, in the next generation, high productivity students switch their choice and attend the less challenging school, leaving low productivity students at the more challenging school. The key reason 
for different behavior of the two groups of students at that stage is that jump in probability of completion (as a result of a switch to the less challenging school) is much higher for the high than for the low productivity students. This seemingly paradoxical situation leads to further reduction of the expected productivity at the more challenging school and to an increase of expected productivity in the less challenging one. As a result, in the next step, low productivity students join high productivity students at the less challenging school. This is the long run outcome.

\section{AN approach}

When employers use adaptive expectations and the students use naïve ones, the situation is essentially like in the RNG case, but with potentially higher number of intermediate steps. Namely, in the first case (illustrated by Fig. 5), for the first two generations all students choose the first university. In the third generation, high productivity students switch to the second one. From the fourth generation on, all students attend the second university. In the second case (Fig. 6) all students enroll into the first school from the first generation.

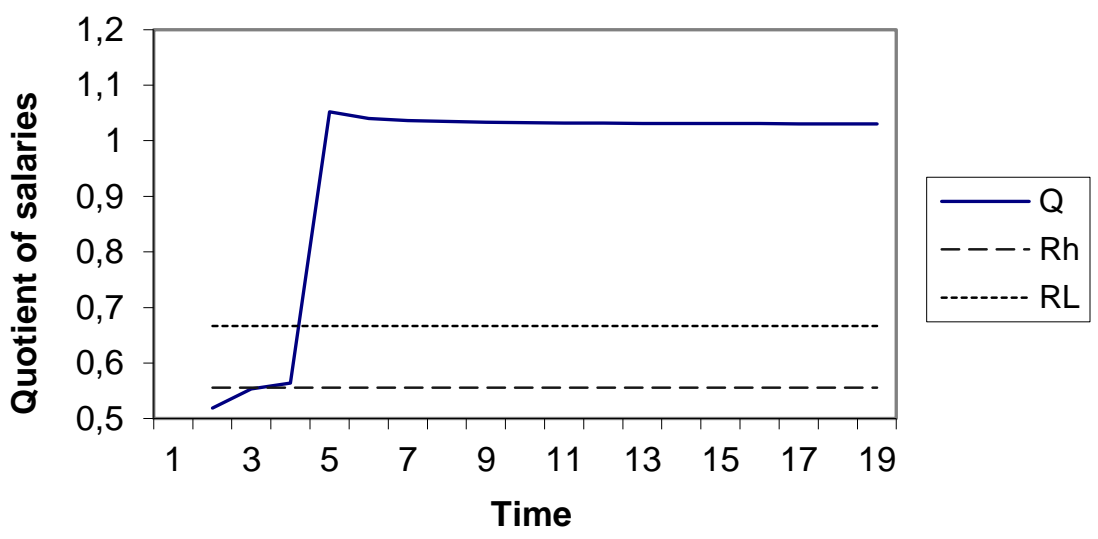

Fig.5

$\mathrm{C}_{1}-\mathrm{C}_{4}-\mathrm{C}_{2}$ dynamics in $\mathrm{AN}$ approach

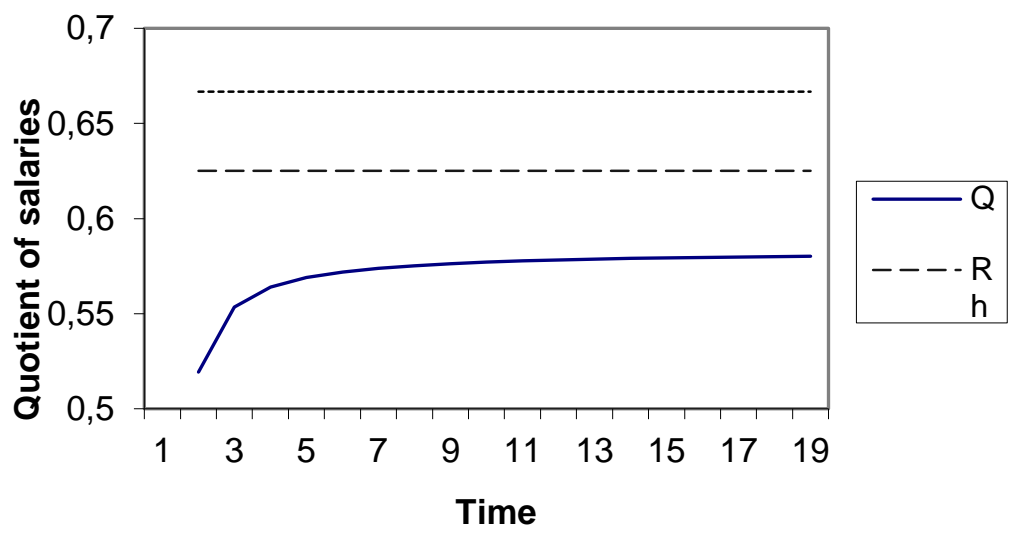

Fig. 6

$\mathrm{C}_{1}$ dynamics in $\mathrm{AN}$ approach 
Example 6.3 In this example we analyze the situation in which long run outcome in RR approach is a mixed equilibrium $M_{1}$. In that equilibrium, high productivity students select more challenging school, while low productivity ones play mixed strategy. Like in Example 6.2, we consider two sets of parameters. They differ from each other only in that in the second case more challenging school increases probability of completion for high productivity students by 5 percentage points. While this change does not influence equilibrium outcome in the RR approach, it leads to very surprising results in the RNG and AN approaches as a consequence of naïve (backward-looking) expectations of students. For the first set of parameters in these two approaches all high productivity students attend the first school in the long run, while low productivity students alternate their choice in different generations $\left(C_{1}-C_{3}\right.$ cycle). On the other hand, and perhaps quite surprisingly, making life a little bit easier for high productivity students at the more challenging school (in terms of probability of completion), ceteris paribus, drives both high and low productivity students away from that school. Moreover, if an increase in probability of completion of the first university for high productivity students is large enough, high productivity students would not switch to the second school.

Table 10

Parameters for Example 6.3 (with lower $p_{H 1}$ )

\begin{tabular}{|c|c|c|c|c|c|c|c|c|c|c|c|}
\hline & \multicolumn{2}{|c|}{$\begin{array}{c}\text { Initial } \\
\text { enrolment }\end{array}$} & \multicolumn{2}{|c|}{ Probabilities } & \multicolumn{2}{|c|}{$\begin{array}{c}\text { Initial } \\
\text { salaries }\end{array}$} & \multirow[t]{2}{*}{$\overline{S_{1}}$} & \multirow[t]{2}{*}{$\bar{S}_{2}$} & \multirow{2}{*}{$\begin{array}{l}\text { Quotient of } \\
\text { probabilities } \\
\left(R_{L} \text { and } R_{H}\right)\end{array}$} & \multicolumn{2}{|c|}{$\begin{array}{c}\text { Distinction } \\
\text { levels }\end{array}$} \\
\hline & $n_{1}$ & $n_{2}$ & $p_{1}$ & $p_{2}$ & $S_{1}(0)$ & $S_{2}(0)$ & & & & $D_{1}$ & $D_{2}$ \\
\hline Low & 80 & 920 & 0.55 & 0.80 & \multirow{2}{*}{1.80} & \multirow{2}{*}{1.24} & \multirow{2}{*}{1.39} & \multirow{2}{*}{1.37} & 0.69 & \multirow{2}{*}{1.27} & \multirow{2}{*}{1.19} \\
\hline High & 250 & 250 & 0.70 & 0.95 & & & & & 0.74 & & \\
\hline
\end{tabular}

Table 11

Parameters for Example 6.3 (with higher $p_{H 1}$ )

\begin{tabular}{|c|c|c|c|c|c|c|c|c|c|c|c|}
\hline & \multicolumn{2}{|c|}{$\begin{array}{c}\text { Initial } \\
\text { enrolment }\end{array}$} & \multicolumn{2}{|c|}{ Probabilities } & \multicolumn{2}{|c|}{$\begin{array}{c}\text { Initial } \\
\text { salaries }\end{array}$} & \multirow[t]{2}{*}{$\bar{S}_{1}$} & \multirow{2}{*}{$\bar{S}_{2}$} & \multirow{2}{*}{$\begin{array}{l}\text { Quotient of } \\
\text { probabilities } \\
\left(R_{L} \text { and } R_{H}\right)\end{array}$} & \multicolumn{2}{|c|}{$\begin{array}{c}\text { Distinction } \\
\text { levels }\end{array}$} \\
\hline & $n_{1}$ & $n_{2}$ & $p_{1}$ & $p_{2}$ & $S_{1}(0)$ & $S_{2}(0)$ & & & & $D_{1}$ & $D_{2}$ \\
\hline Low & 80 & 920 & 0.55 & 0.80 & \multirow{2}{*}{1.81} & \multirow{2}{*}{1.24} & \multirow{2}{*}{1.41} & \multirow{2}{*}{1.37} & 0.69 & \multirow{2}{*}{1.36} & \multirow{2}{*}{1.19} \\
\hline High & 250 & 250 & 0.75 & 0.95 & & & & & 0.79 & & \\
\hline
\end{tabular}

\section{RR approach}

In both cases the equilibrium outcome is mixed state $M_{1}$, which means that all high productivity students select the first school, while low productivity students split their choice. Note that in this example high productivity students have greater expected salary when they alone attend the first school, than when all students attend the second school, 
while low productivity students have greater expected salary when they alone attend the second school, than when all attend the first school. At the same time, the first school is more attractive to low productivity students when only high productivity students attend it. This results in the mixed choice strategy for the low productivity students. Formally, the conditions $Q_{\min }<R_{L}<\frac{S_{L}}{\bar{S}_{1}}$ and $\frac{\bar{S}_{2}}{S_{H}}<R_{H}$ for $M_{1}$ equilibrium are satisfied (Table 1).

\section{RNN approach}

In this case, all students select the second university in each generation. Note that this result differs from the equilibrium in the RR approach which would correspond to the cycle $C_{1}-C_{3}$. Since students have naïve expectations, the initial conditions play important role. In RNN approach, initial enrolment of high and low productivity students is in proportion to their fraction in the general student population. Recall that for a fixed proportion of two types of students enrolled in a school, expected productivity of graduates is a monotonically increasing function of the distinction level of that school. In this example distinction levels are similar at the two schools. This, in turn, results in similar levels of salaries offered to graduates of the two schools. However, since the second school provides a much higher probability of completion for both groups of students, all of them end up choosing the second school.

\section{RNG approach}

When value of $p_{H 1}$ is lower (Table 10) all high productivity students choose the first university in each generation, while low productivity ones alternate between the two choices in consecutive generations. ${ }^{27}$ The mechanism is as follows. After initial enrollments (taken as parameters of the model), in the first generation low productivity students choose the second school while the high productivity ones select the first one. This lowers the expected productivity of graduates of the second school and increases the expected productivity and, thus, attractiveness of graduates of the first school. Now, in the second generation all students select the first school which, in turn, leads to a decrease in the offered salary for graduates of that school, prompting low productivity students to switch to the second school in the third generation. This is then repeated cyclically.

In contrast, in the situation when the first university has higher value of $p_{H 1}$ (Table 11), ceteris paribus, all students will ultimately choose the second university. Thus, quite surprisingly, making life a little bit easier to high productivity students at the more challenging school can drive all students away from that school. This happens for the following reason. Increasing the passing rate of high productivity students at the first school leads to higher expected numbers of high productivity graduates from that school

\footnotetext{
27 This corresponds to the RR approach where, in equilibrium, low productivity students mix their choice in a single generation.
} 
in the initial generation. This, in turn, leads to higher expected productivity and, therefore, higher salary offered for its graduates initially, attracting all students (including low productivity ones) to attend the first school in the next generation. Influx of low productivity students now substantially reduces the salary offered to graduates of the first school in the next step. This drives all students away to the second school starting from the second step. ${ }^{28}$

The difference from the first scenario (Table 10) stems from the fact that in the first step after the initial enrollment there, low productivity students attend the second school (instead of the first one), reducing salary offered to the graduates of the second school (with respect to the initial one) and, thus, making the transition of all students from the first to the second school impossible.

\section{AN approach}

The long run equilibria in this case are the same as in the RNG approach. Dynamics for both sets of parameters is illustrated in Fig. 7 and Fig. 8, respectively. The only important difference with respect to the RNG case is that in the AN approach reputation of universities is adjusting gradually. It may take more than one generation for substantive change of reputation. Despite of the more gradual changes in salaries offered to graduates, due to adaptive nature of the employers' beliefs, these changes are still sufficient for the same mechanisms as in the RNG approach to work. In particular, slightly increasing likelihood of completion for high productivity students at the more challenging school, ceteris paribus, eventually may prove detrimental for the reputation of that school.

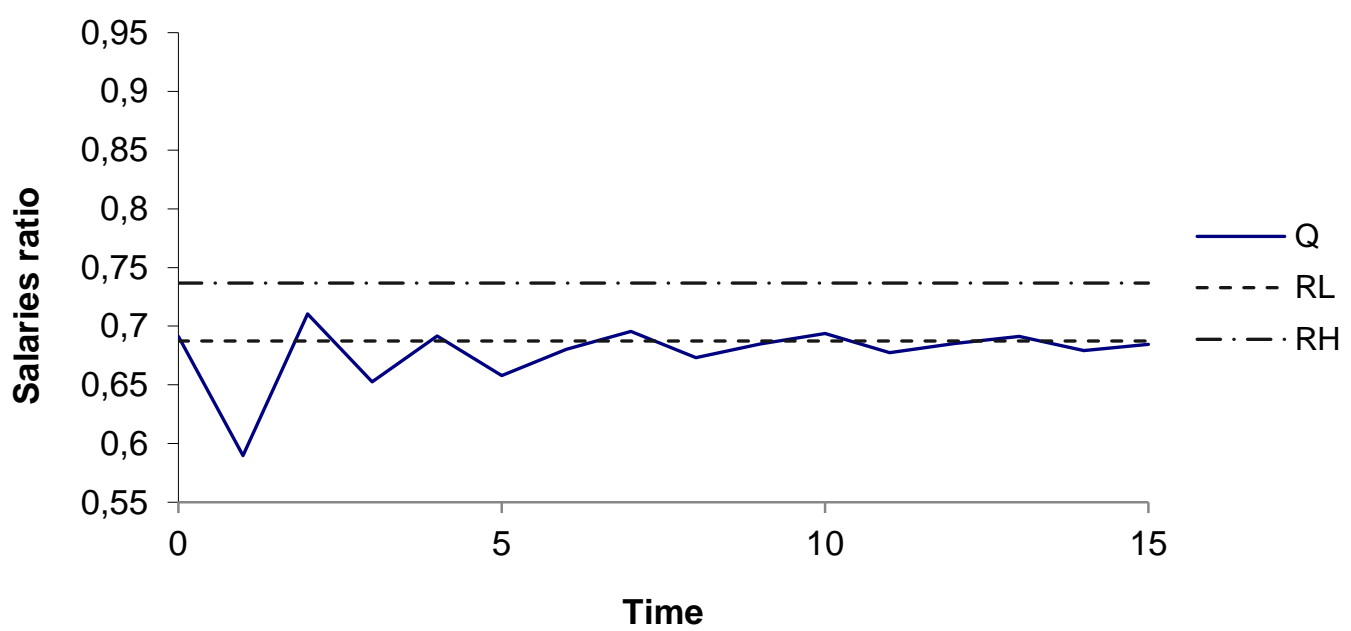

Fig. 7

Cyclic $\mathrm{C}_{1}-\mathrm{C}_{3}$ dynamics in $\mathrm{AN}$ approach

\footnotetext{
${ }^{28}$ The argument assumes that an increase in likelihood of completion is small enough. In case of a large increase in $p_{H 1}$ this would no longer necessarily hold. In that case high productivity students would eventually attend the more challenging school.
} 


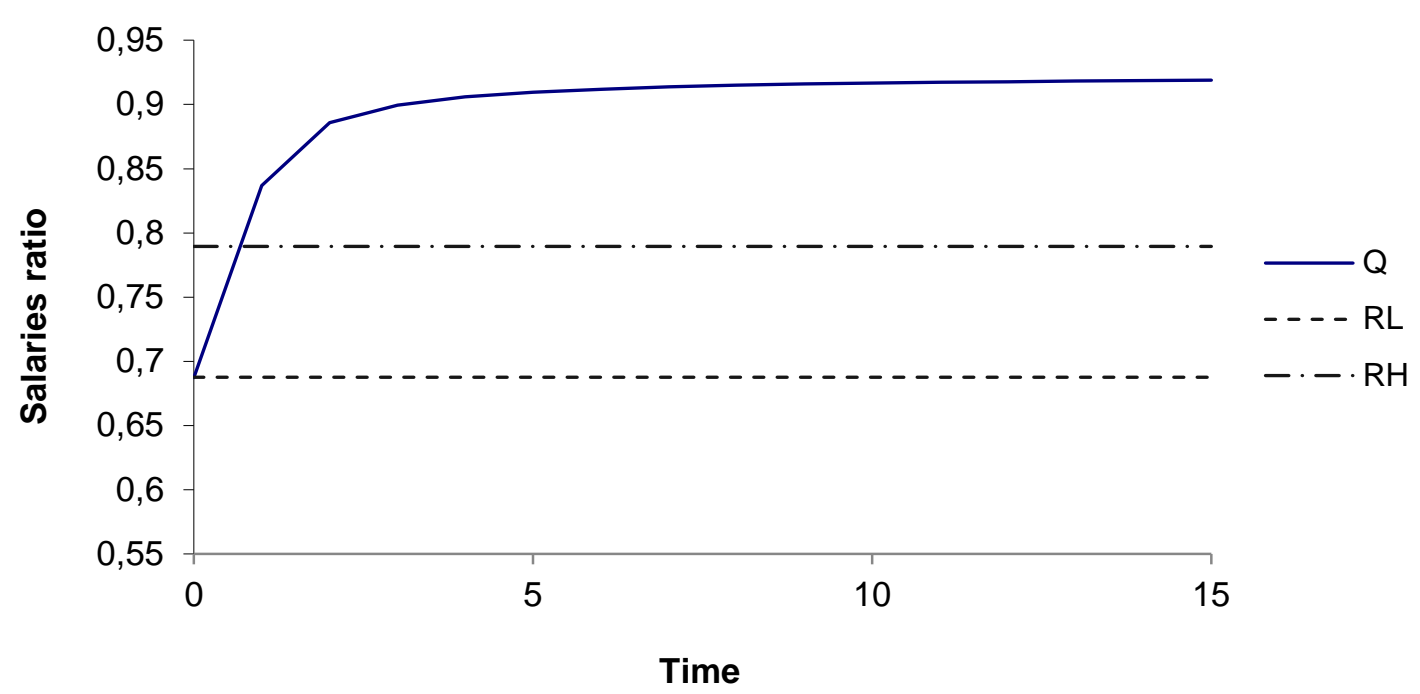

Fig. 8

$\mathrm{C}_{1}-\mathrm{C}_{2}$ dynamics in $\mathrm{AN}$ approach

\section{Concluding remarks}

In this paper we propose a signaling model for determining dynamics of university selection by students. Prospective students of two different productivity types choose between the schools of two different levels of difficulty. They make their enrollment decisions based on their expectations of post-graduation salary. The dynamics is primarily driven by probabilities of completion of each school for the two student types, and by way in which students and employers, who set salaries, form their beliefs. All agents are risk neutral. As far as we know, this is the first theoretical paper that studies how probability of school completion impacts dynamics of university selection in both fully rational and less than fully rational settings.

We find enrollment dynamics when both students and employers are rational, when students are naïve and employers rational and, finally, when students are naïve while employers use adaptive expectations. Regardless of the type of expectations, in the long run three types of equilibria are possible: pooling, separating and a cycle (mixed in case of full rationality). They obtain under very similar general conditions. In addition, long run outcome may depend on the initial enrollment when students are naïve. Relaxing the assumption of rationality typically increases the number of steps (generations of students) needed to reach the steady state. Last but not least, we consider several examples that help us elucidate comparative statics. 
Distinction level, i.e. likelihood ratio of school completion for the two types of students at a given school, plays an important role in determining the nature of long-run outcomes irrespectively of rationality assumptions. In particular, the most natural long-run equilibrium, namely fully separating equilibrium in which high productivity students attend the more challenging while low productivity students attend the less challenging school, can be obtained only when the more challenging school has higher distinction level.

When all agents are rational, steady state is reached in the first generation. In particular, if a university loses all students, it can never regain them in the future. On the other hand, when students are naïve, this may not be the case. In fact, school that at some point runs out of students may in the future even end up with the entire student population. Thus, temporary loss of students does not mean that such school has no long term prospects when agents are not fully rational.

Anecdotal evidence exists that many leading companies prefer to hire people from the most reputable schools. In our model such situation naturally arises when employers use past reputation of schools in order to make predictions about student enrollment distribution, i.e. when they use adaptive expectations. Interestingly, the more challenging school does not necessarily have higher reputation with the employers. Moreover, the reputation of schools may depend, also, on the initial enrollment of students.

Initial enrollment matters when students are naïve. In that case, the initial campaign to attract the best students may be of particular importance. It influences both the reputation of schools and the long run outcome.

An important issue that we address now is how changes in probabilities of completion impact long run outcomes. We show, as expected, that in the case of full rationality making life easier for high productivity students, ceteris paribus, always (at least weakly) increases the number of students at that school. In contrast, when students use naïve expectations, comparative statics can be quite surprising. In particular, it is possible that a slight increase of probability of completion for high productivity students at the more challenging school leads to eventual loss of all students at that school. Thus, an attempt to attract the best students by increasing their probability of completion may sometimes be counterproductive in the long run.

There are several directions of possible future research. In this paper universities are not strategic players. Allowing them to behave strategically may be of particular interest. Also, here we assumed that universities accept all applicants but filter them with differential drop-out rates. This is, essentially, found in practice at many state universities in continental Europe and Latin America, among others. On the other hand, in AngloSaxon countries much of the screening is done at the entering stage. It would be 
important to better understand which of the two systems does a better job in providing the signaling function for the marketplace.

One could try to incorporate some other realistic features into the model. For example, completing a school may not only signal productivity but, also, impact it. The model would be possible to extend to incorporate noise in the student choice (see Feltovich et al (2002), among others). When employers are backward-looking, it may make sense to give more weight to information about recent graduates. These and other interesting issues may be subject of further research. 


\section{References}

Akerlof, G. (1997): "Social Distance and Social Decisions", Econometrica, 65, 10051027.

Alos-Ferrer, C., and Prat, J. (2013): "Job Market Signaling and Employer Learning, Journal of Economic Theory, Vol 148(1), pp. 226-258.

Altonji, J. (1993):“The Demand for and Return to Education When Education Outcomes are Uncertain“, Journal of Labor Economics, 11(1), 48-83.

Amaya, K. (2009): "Perfect Foresight Equilibrium Selection in Signaling Games", MIT Working paper

Banks, J., and J.Sobel (1987):“Equilibrium selection in signaling games,"Econometrica, $55,647-662$.

Broecke, S. (2012), University selectivity and earnings: Evidence from UK data on applications and admissions to university, Economics of Education Review, Vol 31(3), pp. 96-107.

Calvo-Armengol, A., E. Patacchini and Y. Zenou (2009), Peer Effects and Social Networks in Education, Review of Economic Studies, Vol 76(4), pp. 1239-1267.

Cho, I.K., and D. Kreps (1987): "Signaling games and stable equilibria,"Quart. J. Econ., $102,179-221$.

Dellas, H. and P. Sakellaris (2003): "On the Cyclicality of Schooling: Theory and Evidence”, Oxford Economic Papers, 55, 148-172.

Evans, G. W. and S. Honkapohja (2001):Learning and expectations in macroeconomics, Princeton University Press

Farber, H. S., and R. Gibbons (1996):"Learning and Wage Dynamics," The Quarterly Journal of Economics, 111, 1007-1047.

Feltovich,N.R., R. Harbaugh, and T. To (2002):“Too cool for school?Signaling and countersignaling,"Rand J. Econ., 33, 630-649.

Hendel, I., J. Shapiro, and P.Willen (2005): "Educational opportunity and income inequality”, Journal of Public Economics, 89, 841-870 . 
Jacobsen, H.,J., M. Jensen, and B. Sloth (2001): "Evolutionary Learning in Signalling Games", Games and Economic Behavior 34, 34-63

Jovanovic, B. (1979): "Job Matching and the Theory of Turnover",The Journal of Political Economy, 87, 972-990.

Kandori, M., G. Mailath and R. Rob (1993), "Learning, mutation, andlong run equilibria in games,"Econometrica61, 29-56.

Long, M., F. Ferrier and M.Heagney. Stay, play or give it away? Students continuing, changing or leaving university study in first year. Melbourne: Centre for the Economics of Education and Training, 2006.

Mailath, G., M. Okuno-Fujiwara and A. Postlewaite (1993), "Beliefbasedrefinements in signallinggames,"Journal of Economic Theory 60, 241-276.

Manski, C. (1993), “Adolescent Econometricians: How Do Youth Infer the Returnsto Schooling?" (1993),. Published in: Studies of Supply and Demand in Higher Education, University of Chicago Press, C. Clotfelter and M. Rothschild, editors, 43-60.

Manski, C. (1989), "Schooling as Experimentation: a Reappraisal of the Postsecondary Dropout Phenomenon“, Economics of Education Review,8(4), 305-312

Mas-Colell, A, Whinston, M. D. and Green, J. R (1995), "Microeconomic Theory," Oxford University Press

Matsui, A. and K. Matsuyama (1995), “An approach to equilibriumselection,"Journal of Economic Theory 65, 415-443.

Noldeke, G., and L. Samuelson (1997): “A Dynamic Model of Equilibrium Selection in Signaling Markets”, Journal of Economic Theory, 73, 118-156.

Noldeke, G., and E. Van Damme (1990):"Signalling in a dynamic labourmarket,"Rev. Econ. Stud., 57, 1-23.

OECD(2007), Education at a Glance: OECD Indicators - 2007 Edition, OECD, Paris.

OECD(2010), Education at a Glance: OECD Indicators - 2010 Edition, OECD, Paris.

Riley, J. G. (2001): "Silver Signals: Twenty-Five Years of Screening and Signaling,"Journalof Economic Literature, 39, 432-478. 
Riley, J. G. (1979), “Informational Equilibrium”, Econometrica, 47, 331-359.

Spence, A. M. (1973):“Job market signaling,”Quart. J. Econ., 87, 355-374.

Spence, A. M. (1974): Market signaling, information transfer in hiring and related processes, Harvard University Press, Cambridge.

Swinkels, J. M. (1999): "Education signalling with preemptive offers",Rev. Econ. Stud., 66, 949-970.

Weiss, A. (1983): “A Sorting-cum-learning Model of Education”, Journal of Political Economy, 91, 420-442.

Wiswall, M, and B. Zafar (2011), "Belief Updating among College Students: Evidence from Experimental Variation“, Federal Reserve Bank of New York Staff Reports, no. 516

Zafar, B. (2011), "How Do College Students Form Expectations?", Journal of Labor Economics, 2, 301-338 


\section{Appendix}

\section{Proof of Proposition 2.1}

From definition of $\overline{S_{1}}$ we have

$$
\begin{gathered}
\bar{S}_{1}=\frac{n_{H} p_{H 1}}{n_{H} p_{H 1}+n_{L} p_{L 1}} S_{H}+\frac{n_{L} p_{L 1}}{n_{H} p_{H 1}+n_{L} p_{L 1}} S_{L}=\frac{n_{H} R_{H} p_{H 2}}{n_{H} R_{H} p_{H 2}+n_{L} R_{L} p_{L 2}} S_{H}+\frac{n_{L} R_{L} p_{L 2}}{n_{H} R_{H} p_{H 2}+n_{L} R_{L} p_{L 2}} S_{L}= \\
=\frac{n_{H} p_{H 2}}{n_{H} p_{H 2}+n_{L} p_{L 2}} S_{H}+R \frac{n_{L} p_{L 2}}{n_{H} p_{H 2}+n_{L} p_{L 2}} S_{L} .
\end{gathered}
$$

Using calculus one can easily see that function $f$ defined as

$$
f(x)=\frac{n_{H} p_{H 2}}{n_{H} p_{H 2}+n_{L} p_{L 2} x} S_{H}+x \frac{n_{L} p_{L 2}}{n_{H} p_{H 2}+n_{L} p_{L 2} x} S_{L}
$$

is a decreasing function of $x$, and so $R>1$, is equivalent to $f(R)<f(1)$ i.e. to

$$
\bar{S}_{1}<\frac{n_{H} p_{H 2}}{n_{H} p_{H 2}+n_{L} p_{L 2}} S_{H}+\frac{n_{L} p_{L 2}}{n_{H} p_{H 2}+n_{L} p_{L 2}} S_{L}=\bar{S}_{2}
$$

Q.E.D.

\section{Proof of Proposition 3.1}

We will analyze all possible states and conditions when they give rise to PBE. If students use the strategies $M_{2}$ or $C_{4}$, low productivity students get a greater payoff if they move to the second university, while if the strategy $M_{3}$ is used high productivity students have a strictly greater payoff at the second university. We do not consider the strategy $M_{5}$, in which PBE can only arise in the non-generic case $R_{L}=R_{H}$. Namely, when high productivity students split their choices in PBE then their payoffs have to be the same for the two universities, which means that the salaries ratio $Q$ is equal to $R_{H}$. Similarly, low productivity students split their choices in PBE when $Q$ is equal to $R_{L}$.

To analyze the other possible strategies, we first compute payoffs, and summarize them in the Table 1. The payoffs are computed in a straightforward way, bearing in mind that in $\operatorname{PBE} Q$ is equal to $R_{H}$ when high productivity students split their choices, and to $R_{L}$ when low productivity students do so. 
When all students of a given type choose the same university, in PBE that choice has to have the better payoff than the payoff at the other university. If no students go to the other university, as is the case for $C_{1}$ and $C_{2}$, the salary offered at the other university depends on the beliefs held by employers, and can take any value between $S_{L}$ and $S_{H}$. If there is a belief i.e. the salary level in that range which makes the student payoff lower in the unused university, we will have a PBE. Taking this into account, one can derive the conditions listed in the proposition.

Q.E.D.

\section{Proof of Theorem 3.1}

When $R_{H}<\frac{\bar{S}_{2}}{S_{H}}$ then high productivity students have a choice of the second university strictly dominating the choice of the first university, making $C_{2}$ a preferred PBE. This always eliminates $M_{4}$, which can only occur when $R_{H}<\frac{\bar{S}_{2}}{S_{H}}$.

If $R>1$, then $C_{2}$ will always be preferred by the D1 divinity condition. Namely, in equilibrium $C_{1}$ the first to deflect to the second university will be the high productivity students, and hence the D1 belief is such that salary on the second university is $S_{H}$, and since the second university is has greater probabilities of completion it also has the greater payoffs, hence $C_{1}$ is eliminated.

If $R<1$, from conditions listed in Proposition 3.1. it is clear that PBEs $C_{1}, C_{3}$ and $M_{1}$ never occur together. Thus, we have to separate them from $C_{2}$. If $R_{H}<\frac{\bar{S}_{2}}{S_{H}}$ we have already seen that $C_{2}$ is the preferred equilibrium by dominance. On the other side, if $R_{H}>\frac{\bar{S}_{2}}{S_{H}}$ testing for D1 eliminates $C_{2}$ when $R<1$, since then the high productivity students are the first to deflect to the first university, and the D1 belief is such that salary on the first university is $S_{H}$, making the first university the preferred choice for the high productivity students.

Putting these conditions together with the ones from Proposition 3.1 gives the desired result.

Q.E.D. 


\section{Proof of Theorem 4.2}

Table A.1

State sequences for $R>1$ in $\mathrm{RNG}$ approach

\begin{tabular}{|c|c|c|c|c|c|c|c|c|}
\hline \multirow{3}{*}{$\begin{array}{c}\text { Long run } \\
\text { equilibrium }\end{array}$} & \multirow{3}{*}{ Case } & \multicolumn{6}{|c|}{ Dynamics } & \multirow{3}{*}{ Conditions } \\
\hline & & \multicolumn{2}{|c|}{$t=1$} & \multicolumn{2}{|c|}{$t=2$} & \multicolumn{2}{|c|}{$t=3$} & \\
\hline & & State & $Q$ & State & $Q$ & State & $Q$ & \\
\hline$C_{1}$ & 1 & $C_{1}$ & $\frac{S_{2}(1)}{\bar{S}_{1}}$ & $C_{1}$ & $\frac{S_{2}(1)}{\bar{S}_{1}}$ & $C_{1}$ & $\frac{S_{2}(1)}{\bar{S}_{1}}$ & $\frac{S_{2}(1)}{\bar{S}_{1}}<R_{H}$ \\
\hline \multirow{4}{*}{$C_{2}$} & 2 & $C_{2}$ & $\frac{\bar{S}_{2}}{S_{1}(1)}$ & $C_{2}$ & $\frac{\bar{S}_{2}}{S_{1}(1)}$ & $C_{2}$ & $\frac{\bar{S}_{2}}{S_{1}(1)}$ & $Q(1)>R_{L}$ \\
\hline & 3 & $C_{1}$ & $\frac{S_{2}(1)}{\bar{S}_{1}}$ & $C_{2}$ & $\frac{\bar{S}_{2}}{\bar{S}_{1}}$ & $C_{2}$ & $\frac{\bar{S}_{2}}{\bar{S}_{1}}$ & $Q(1)<R_{H}, \frac{S_{2}(1)}{\bar{S}_{1}}>R_{L}$ \\
\hline & 4 & $C_{1}$ & $\frac{S_{2}(1)}{\bar{S}_{1}}$ & $C_{4}$ & $\frac{S_{H}}{S_{L}}$ & $C_{2}$ & $\frac{\bar{S}_{2}}{S_{L}}$ & $\begin{array}{c}Q(1)<R_{H} \\
R_{H}<\frac{S_{2}(1)}{\bar{S}_{1}}<R_{L}\end{array}$ \\
\hline & 5 & $C_{4}$ & $\frac{S_{H}}{S_{L}}$ & $C_{2}$ & $\frac{\bar{S}_{2}}{S_{L}}$ & $C_{2}$ & $\frac{\bar{S}_{2}}{S_{L}}$ & $R_{H}<Q(1)<R_{L}$ \\
\hline
\end{tabular}

(Last state is repeated indefinitely)

Using the assumption that employer makes perfect prediction of productivity of graduates in offering salary, while keeping previous level of salary for university in which no students enroll at a given time step, we can deduce the salaries in the next time step. We summarize them in the Table A.3

If the system is initially in state $C_{1}$ and $Q(1)=\frac{S_{2}(1)}{\bar{S}_{1}}<\min \left\{R_{H}, R_{L}\right\}$ the system will remain in this state indefinitely. Similarly, if the system is initially in state $C_{2}$ and $Q(1)=\frac{\bar{S}_{2}}{S_{1}(1)}>\max \left\{R_{H}, R_{L}\right\}$ the system will remain in state $C_{2}$ indefinitely. Otherwise, if we are initially in one of these two states, a change of state will occur, and the salaries will be determined by the last two states. Also, salaries do not depend on initial conditions if we start in states $C_{3}$ or $C_{4}$.

Bearing this in mind, and depending on the values of the parameters of the system, we get sequences of first few states, which are together with corresponding salaries summarized in Tables A.1 and A.2. After first 3 states, either the last state, or the cycle of the last two states, is repeated indefinitely.

Q.E.D. 
Table A. 2

State sequences for $R<1$ in $\mathrm{RNG}$ approach

\begin{tabular}{|c|c|c|c|c|c|c|c|c|}
\hline \multirow{3}{*}{$\begin{array}{l}\text { Long run } \\
\text { equilibria }\end{array}$} & \multirow{3}{*}{ Case } & \multicolumn{6}{|c|}{ Dynamics } & \multirow{3}{*}{ Conditions } \\
\hline & & \multicolumn{2}{|c|}{$t=1$} & \multicolumn{2}{|c|}{$t=2$} & \multicolumn{2}{|c|}{$t=3$} & \\
\hline & & State & $Q$ & State & $Q$ & State & $Q$ & \\
\hline \multirow{5}{*}{$C_{1}$} & 1 & $C_{1}$ & $\frac{S_{2}(1)}{\bar{S}_{1}}$ & $C_{1}$ & $\frac{S_{2}(1)}{\bar{S}_{1}}$ & $C_{1}$ & $\frac{S_{2}(1)}{\bar{S}_{1}}$ & $Q(1)<R_{L}, \frac{S_{2}(1)}{\bar{S}_{1}}<R_{L}$ \\
\hline & 2 & $C_{2}$ & $\frac{\bar{S}_{2}}{S_{1}(1)}$ & $C_{1}$ & $\frac{\bar{S}_{2}}{\bar{S}_{1}}$ & $C_{1}$ & $\frac{\bar{S}_{2}}{\bar{S}_{1}}$ & $Q(1)>R_{H}, \frac{\bar{S}_{2}}{S_{1}(1)}<R_{L}$ \\
\hline & 3 & $C_{2}$ & $\frac{\bar{S}_{2}}{S_{1}(1)}$ & $C_{3}$ & $\frac{S_{L}}{S_{H}}$ & $C_{1}$ & $\frac{S_{L}}{\bar{S}_{1}}$ & $Q(1)>R_{H}, \frac{S_{L}}{\bar{S}_{1}}<R_{L}, R_{L}<\frac{\bar{S}_{2}}{S_{1}(1)}<R_{H}$ \\
\hline & 4 & $C_{3}$ & $\frac{S_{L}}{S_{H}}$ & $C_{1}$ & $\frac{S_{L}}{\bar{S}_{1}}$ & $C_{1}$ & $\frac{S_{L}}{\bar{S}_{1}}$ & $R_{L}<Q(1)<R_{H}, \frac{S_{L}}{\bar{S}_{1}}<R_{L}$ \\
\hline & 5 & $C_{1}$ & $\frac{S_{2}(1)}{\bar{S}_{1}}$ & $C_{3}$ & $\frac{S_{L}}{S_{H}}$ & $C_{1}$ & $\frac{S_{L}}{\bar{S}_{1}}$ & $Q(1)<R_{L}, \frac{S_{L}}{\bar{S}_{1}}<R_{L}, R_{L}<\frac{S_{2}(1)}{\bar{S}_{1}}<R_{H}$ \\
\hline \multirow{3}{*}{$C_{2}$} & 6 & $C_{2}$ & $\frac{\bar{S}_{2}}{S_{1}(1)}$ & $C_{2}$ & $\frac{\bar{S}_{2}}{S_{1}(1)}$ & $C_{2}$ & $\frac{\bar{S}_{2}}{S_{1}(1)}$ & $Q(1)>R_{H}, \frac{\bar{S}_{2}}{S_{1}(1)}>R_{H}$ \\
\hline & 7 & $C_{1}$ & $\frac{S_{2}(1)}{\bar{S}_{1}}$ & $C_{2}$ & $\frac{\bar{S}_{2}}{\bar{S}_{1}}$ & $C_{2}$ & $\frac{\bar{S}_{2}}{\bar{S}_{1}}$ & $Q(1)<R_{L}, \frac{S_{2}(1)}{\bar{S}_{1}}>R_{H}$ \\
\hline & 8 & $C_{3}$ & $\frac{S_{L}}{S_{H}}$ & $C_{1}$ & $\frac{S_{L}}{\bar{S}_{1}}$ & $C_{2}$ & $\frac{\bar{S}_{2}}{\bar{S}_{1}}$ & $R_{L}<Q(1)<R_{H}, Q_{\min }<R_{L}, \frac{S_{L}}{\bar{S}_{1}}>R_{H}$ \\
\hline \multirow{2}{*}{$C_{3}$} & 9 & $C_{3}$ & $\frac{S_{L}}{S_{H}}$ & $C_{3}$ & $\frac{S_{L}}{S_{H}}$ & $C_{3}$ & $\frac{S_{L}}{S_{H}}$ & $Q(1)<R_{H}, Q_{\min }>R_{L}$ \\
\hline & 10 & $C_{2}$ & $\frac{\bar{S}_{2}}{S_{1}(1)}$ & $C_{3}$ & $\frac{S_{L}}{S_{H}}$ & $C_{3}$ & $\frac{S_{L}}{S_{H}}$ & $Q(1)>R_{H}, Q_{\min }>R_{L}, \frac{\bar{S}_{2}}{S_{1}(1)}<R_{H}$ \\
\hline \multirow{3}{*}{$\begin{array}{c}C_{1}-C_{3} \\
\text { cycle }\end{array}$} & 11 & $C_{1}$ & $\frac{S_{2}(1)}{\bar{S}_{1}}$ & $C_{3}$ & $\frac{S_{L}}{S_{H}}$ & $C_{1}$ & $\frac{S_{L}}{\bar{S}_{1}}$ & $Q(1)<R_{L}, \frac{S_{L}}{\bar{S}_{1}}>R_{L}, \frac{S_{2}(1)}{\bar{S}_{1}}<R_{H}$ \\
\hline & 12 & $C_{3}$ & $\frac{S_{L}}{S_{H}}$ & $C_{1}$ & $\frac{S_{L}}{\bar{S}_{1}}$ & $C_{3}$ & $\frac{S_{L}}{S_{H}}$ & $\begin{array}{c}R_{L}<Q(1)<R_{H}, Q_{\min }<R_{L} \\
R_{L}<\frac{S_{L}}{\bar{S}_{1}}<R_{H}\end{array}$ \\
\hline & 13 & $C_{2}$ & $\frac{\bar{S}_{2}}{S_{1}(1)}$ & $C_{3}$ & $\frac{S_{L}}{S_{H}}$ & $C_{1}$ & $\frac{S_{L}}{\bar{S}_{1}}$ & $\begin{array}{c}Q(1)>R_{H}, \frac{\bar{S}_{2}}{S_{1}(1)}<R_{H}, Q_{\mathrm{min}}<R_{L} \\
R_{L}<\frac{S_{L}}{\bar{S}_{1}}\end{array}$ \\
\hline
\end{tabular}

(Last state is repeated indefinitely except for the $C_{1}-C_{3}$ cycle, where the system alternates between the two states indefinitely) 
Table A.3

States and payoffs in $\mathrm{RN}$ approach

\begin{tabular}{lllll} 
& \multicolumn{4}{c}{ State at time $t$} \\
\cline { 2 - 5 } & $C_{1}$ & $C_{2}$ & $C_{3}$ & $C_{4}$ \\
\hline$S_{1}(t+1)$ & $\bar{S}_{1}$ & $S_{1}(t)$ & $S_{H}$ & $S_{L}$ \\
\hline$S_{2}(t+1)$ & $S_{2}(t)$ & $\bar{S}_{2}$ & $S_{L}$ & $S_{H}$ \\
\hline
\end{tabular}

\section{Proof of Proposition 5.1}

Let us show that it is impossible to have $\bar{S}_{1}<S_{1}(t)$ and $\bar{S}_{2}<S_{2}(t)$. These conditions are equivalent to $\frac{N_{H 1}(t-1)}{N_{L 1}(t-1)}>\frac{N_{H}(t-1)}{N_{L}(t-1)}$ and $\frac{N_{H 2}(t-1)}{N_{L 2}(t-1)}>\frac{N_{H}(t-1)}{N_{L}(t-1)}$, which is contrary to $N_{H 1}(t-1)+N_{H 2}(t-1)=N_{H}(t-1)$ and $N_{L 1}(t-1)+N_{L 2}(t-1)=N_{L}(t-1)$. Similarly, one can see that it is not possible to have $\bar{S}_{1}>S_{1}(t)$ and $\bar{S}_{2}>S_{2}(t)$. The exclusion of these two cases proves the proposition.

Q.E.D.

\section{Proof of Theorem 5.1}

If the system is in state $C_{1}$, then $Q(t)<R_{H}$. If we remain in the same state, everyone will enroll in $U_{1}$, and consequently $S_{2}(t)$ will not change, and $S_{1}(t)$ will move towards $\bar{S}_{1}$.

If $S_{1}(t)>\bar{S}_{1}$, then $S_{1}(t)>S_{1}(t+1)>\bar{S}_{1}$ and hence $Q(t)<Q(t+1)$. By Proposition 5.1, $\bar{S}_{2} \geq S_{2}(t+1)$, and we have $Q(t)<Q(t+1)<\frac{S_{2}(t+1)}{\bar{S}_{1}} \leq \frac{\bar{S}_{2}}{\bar{S}_{1}}=\bar{Q}$. The case $S_{1}(t)<\bar{S}_{1}$ is considered analogously.

If the system is in state $C_{2}$, then $Q(t)>R_{L}$. As long as we remain in this state, everyone enrolls in $U_{2}$, so $S_{1}(t)$ does not change, while $S_{2}(t)$ moves towards $\bar{S}_{2}$, since ratio of number of alumni students of the two types approaches the ratio of the total number of students of the two types.

If $S_{2}(t)>\bar{S}_{2}$, then $S_{2}(t)>S_{2}(t+1)>\bar{S}_{2}$ and hence $Q(t)>Q(t+1)$. By Proposition 5.1, $\bar{S}_{1} \geq S_{1}(t+1)$, andwe have $Q(t)>Q(t+1)>\frac{\bar{S}_{2}}{S_{1}(t+1)} \geq \frac{\bar{S}_{2}}{\bar{S}_{1}}=\bar{Q}$. The case $S_{2}(t)<\bar{S}_{2}$ is considered analogously. 
If the system is in state $C_{3}$ then $S_{1}(t)$ moves towards $S_{H}$, while $S_{2}(t)$ moves towards $S_{L}$, and hence $Q(t)$ moves towards $Q_{\min }$.

If the system is in state $C_{4}$ then $S_{1}(t)$ moves towards $S_{L}$, while $S_{2}(t)$ moves towards $S_{h}$, and hence $Q(t)$ moves towards $Q_{\max }$.

Q.E.D.

\section{Proof of Theorem 5.2}

Table A.4

State sequences for $R>1$ in AN approach

\begin{tabular}{|c|c|c|c|}
\hline $\begin{array}{l}\text { Long run } \\
\text { equilibrium }\end{array}$ & Case & Dynamics & Conditions \\
\hline$C_{1}$ & 1 & $C_{1} \rightarrow C_{1} \rightarrow \cdots$ & $\frac{S_{2}(1)}{\bar{S}_{1}}<R_{H}$ \\
\hline \multirow{5}{*}{$C_{2}$} & 2 & $C_{2} \rightarrow C_{2} \rightarrow \cdots$ & $Q(1)>R_{L}$ \\
\hline & 3 & $C_{1} \rightarrow \cdots \rightarrow C_{1} \rightarrow C_{2} \rightarrow C_{2} \rightarrow \cdots$ & $\begin{array}{l}Q(1)<R_{H} \\
\frac{S_{2}(1)}{\bar{S}_{1}}>R_{L} \\
\text { and not }(*)\end{array}$ \\
\hline & $4 \mathrm{a}$ & $C_{1} \rightarrow \cdots \rightarrow C_{1} \rightarrow C_{4} \rightarrow \cdots \rightarrow C_{4} \rightarrow C_{2} \rightarrow C_{2} \rightarrow \cdots$ & $\begin{array}{l}Q(1)<R_{H} \\
\frac{S_{2}(1)}{\bar{S}_{1}}>R_{L} \\
\text { and (*) }\end{array}$ \\
\hline & $4 b$ & $C_{1} \rightarrow \cdots \rightarrow C_{1} \rightarrow C_{4} \rightarrow \cdots \rightarrow C_{4} \rightarrow C_{2} \rightarrow C_{2} \rightarrow \cdots$ & $\begin{array}{c}Q(1)<R_{H}, \\
R_{H}<\frac{S_{2}(1)}{\bar{S}_{1}}<R_{L}\end{array}$ \\
\hline & 5 & $C_{4} \rightarrow \cdots C_{4} \rightarrow C_{2} \rightarrow C_{2} \rightarrow \cdots$ & $R_{H}<Q(1)<R_{L}$ \\
\hline
\end{tabular}

Condition (*): There is an integer in the interval $\left(\frac{\mu_{1}(0)}{\bar{\mu}_{1}} \cdot \frac{R_{H}: Q(1)-1}{1-R_{H}: \frac{S_{2}(1)}{\bar{S}_{1}}}, \frac{\mu_{1}(0)}{\bar{\mu}_{1}} \cdot \frac{R_{L}: Q(1)-1}{1-R_{L}: \frac{S_{2}(1)}{\bar{S}_{1}}}\right)$

where $\mu_{1}(0)$ and $\mu_{2}(0)$ denote the expected number of graduates who enrolled the first and the second university in initial time, respectively, and $\bar{\mu}_{1}$ and $\bar{\mu}_{2}$ denote the expected number of graduates from the first and the second university when all students respectively choose the first and the second university. Case $R>1$ : 
Table A.5

State sequences for $R<1$ in AN approach

\section{Long run}

equilibria

3b $\quad C_{2} \rightarrow \cdots \rightarrow C_{2} \rightarrow E \rightarrow \cdots \rightarrow E \rightarrow C_{1} \rightarrow C_{1} \rightarrow \cdots$

\begin{tabular}{ccc}
\hline $4 \rightarrow \cdots \rightarrow E \rightarrow C_{1} \rightarrow C_{1} \rightarrow \cdots$ & $R_{L}<Q(1)<R_{H}, \frac{S_{L}}{\bar{S}_{1}}<R_{L}$ \\
\hline 5 & $D \rightarrow \cdots \rightarrow D \rightarrow C_{1} \rightarrow C_{1} \rightarrow \cdots$ & $Q(1)<R_{L}, \frac{S_{L}}{\bar{S}_{1}}<R_{L}, R_{L}<\frac{S_{2}(1)}{\bar{S}_{1}}<R_{H}$ and not $(* * *)$ \\
\hline$C_{2} \rightarrow C_{2} \rightarrow \cdots$ & $Q(1)>R_{H}, \frac{\bar{S}_{2}}{S_{1}(1)}>R_{H}$
\end{tabular}

\section{Dynamics}

$\begin{array}{clc}C_{1} \rightarrow C_{1} \rightarrow \cdots & Q(1)<R_{L}, \frac{S_{2}(1)}{\bar{S}_{1}}<R_{L} \\ 2 \quad C_{2} \rightarrow \cdots \rightarrow C_{2} \rightarrow C_{1} \rightarrow C_{1} \rightarrow \cdots & Q(1)>R_{H}, \frac{\bar{S}_{2}}{S_{1}(1)}<R_{L} \text { and not (**) }\end{array}$

3a $\quad C_{2} \rightarrow \cdots \rightarrow C_{2} \rightarrow E \rightarrow \cdots \rightarrow E \rightarrow C_{1} \rightarrow C_{1} \rightarrow \cdots$

$$
C_{1} \rightarrow \cdots \rightarrow C_{1} \rightarrow C_{2} \rightarrow C_{2} \rightarrow \cdots
$$

$E \rightarrow \cdots \rightarrow E \rightarrow C_{2} \rightarrow C_{2} \rightarrow \cdots$

$C_{2}$

$8 \mathrm{~b}$

9a $\quad C_{1} \rightarrow \cdots \rightarrow C_{1} \rightarrow E \rightarrow \cdots \rightarrow E \rightarrow C_{2} \rightarrow C_{2} \rightarrow \cdots$

$9 b$

10

$C_{3}$

11

$$
C_{1} \rightarrow \cdots \rightarrow C_{1} \rightarrow E \rightarrow \cdots \rightarrow E \rightarrow C_{2} \rightarrow C_{2} \rightarrow \cdots
$$

$$
C_{3} \rightarrow C_{3} \rightarrow \cdots
$$

$C_{2} \rightarrow \cdots \rightarrow C_{2} \rightarrow C_{3} \rightarrow C_{3} \rightarrow \cdots$

$C_{1}-C_{3}$ cycle

$$
\begin{gathered}
Q(1)<R_{L}, \frac{S_{2}(1)}{\bar{S}_{1}}>R_{H} \text { and not (*) } \\
R_{L}<Q(1)<R_{H}, Q_{\mathrm{min}}<R_{L}, \frac{S_{L}}{\bar{S}_{1}}>R_{H}
\end{gathered}
$$$$
R_{L}<Q(1)<R_{H}, Q_{\mathrm{min}}<R_{L}, R_{L}<\frac{S_{L}}{\bar{S}_{1}}<R_{H} \text { and (***) }
$$$$
Q(1)<R_{L}, \frac{S_{2}(1)}{\bar{S}_{1}}>R_{H} \text { and }(*)
$$$$
Q(1)<R_{L}, R_{L}<\frac{S_{2}(1)}{\bar{S}_{1}}<R_{H} \text { and }(* * *)
$$$$
Q(1)<R_{H}, Q_{\text {min }}>R_{L}
$$$$
Q(1)>R_{H}, Q_{\min }>R_{L}, \frac{\bar{S}_{2}}{S_{1}(1)}<R_{H}
$$

$$
\begin{array}{cc}
D \rightarrow D \rightarrow \cdots & Q(1)<R_{L}, \frac{S_{L}}{\bar{S}_{1}}>R_{L}, \frac{S_{2}(1)}{\bar{S}_{1}}<R_{H} \text { and not (***) } \\
E \rightarrow E \rightarrow \cdots & R_{L}<Q(1)<R_{H}, Q_{\min }<R_{L}, R_{L}<\frac{S_{L}}{\bar{S}_{1}}<R_{H} \text { and not (**) } \\
C_{2} \rightarrow \cdots \rightarrow C_{2} \rightarrow E \rightarrow E \rightarrow \cdots & Q(1)>R_{H}, \frac{\bar{S}_{2}}{S_{1}(1)}<R_{H}, Q_{\text {min }}<R_{L}, R_{L}<\frac{S_{L}}{\bar{S}_{1}}
\end{array}
$$

12

13

14 
Here $\quad D$ is a sequence $\quad C_{1} \rightarrow \cdots \rightarrow C_{1} \rightarrow C_{3} \rightarrow \cdots \rightarrow C_{3} \quad$ and $E$ a sequence $C_{3} \rightarrow \cdots \rightarrow C_{3} \rightarrow C_{1} \rightarrow \cdots \rightarrow C_{1}$

Condition (**): There is an integer in the interval $\left(\frac{\mu_{2}(0)}{\bar{\mu}_{2}} \cdot \frac{Q(1)-R_{H}}{R_{H}-\frac{\bar{S}_{2}}{S_{1}(1)}}, \frac{\mu_{2}(0)}{\bar{\mu}_{2}} \cdot \frac{Q(1)-R_{L}}{R_{L}-\frac{\bar{S}_{2}}{S_{1}(1)}}\right)$

Condition (***): There are positive integers $m$ and $k$ such that

$\frac{\left(S_{2}(1) \mu_{2}(0)+S_{L} m n_{L} p_{L 2}\right)\left(\mu_{1}(0)+m n_{H} p_{H 1}+(k-1) \bar{\mu}_{1}\right)}{\left(\mu_{2}(0)+m n_{L} p_{L 2}\right)\left(\mu_{1}(0) S_{1}(1)+S_{H} m n_{H} p_{H 1}+\bar{S}_{1}(k-1) \bar{\mu}_{1}\right)}<R_{L}$ and $\frac{\left(S_{2}(1) \mu_{2}(0)+S_{L} m n_{L} p_{L 2}\right)\left(\mu_{1}(0)+m n_{H} p_{H 1}+k \bar{\mu}_{1}\right)}{\left(\mu_{2}(0)+m n_{L} p_{L 2}\right)\left(\mu_{1}(0) S_{1}(1)+S_{H} m n_{H} p_{H 1}+\bar{S}_{1} k \bar{\mu}_{1}\right)}>R_{H}$

Since $R>1$, we have $R_{H}<R_{L}<1<\bar{Q}<Q_{\max }$. Using Theorem 5.1 and our definition of states from Table 3, we see that if $R_{H}<Q(t)<R_{L}$ then $Q(t)$ moves towards $Q_{\max }$. If the system would remain in state $C_{4}$, then $Q(t)$ would converge to $Q_{\max }$, as the salaries would converge to the two extreme values. But $R_{L}<Q_{\max }$, so the system has to leave the state $C_{4}$ and enter state $C_{2}$. So, $Q(t)$ starts moving towards $\bar{Q}$, but not necessary converging to it. Thus, if we start from state $C_{2}$ or $C_{4}$, we end up in state $C_{2}$, according to conditions in Table A.1, with possible repetition of states.

If the initially system is in state $C_{1}$, then $S_{2}$ doesn't change over time as long as we remain in $C_{1}$, while $S_{1}$ converges to $\bar{S}_{1}$. Thus, if $\frac{S_{2}(1)}{\bar{S}_{1}}<R_{H}$, the system will remain in $C_{1}$ indefinitely. If $R_{H}<\frac{S_{2}(1)}{\bar{S}_{1}}<R_{L}$, the system will enter the transient state $C_{4}$ and eventually end up in $C_{2}$. If $\frac{S_{2}(1)}{\bar{S}_{1}}>R_{L}$, weather the system enters thestate $C_{4}$ or not after a certain number of steps will depend on the additional condition (*), which can be derived using elementary algebra. Again, the system ends up in $C_{2}$.

Bearing this in mind, we obtain possibilities listed in Table A.1.

Case $R<1$

Using Theorem 5.1 and our definition of states from Table 1, we see that if $R_{L}<Q(t)<R_{H}$ then $Q(t)$ moves towards $Q_{\text {min }}$. If $Q_{\mathrm{min}} \in\left(R_{L}, R_{H}\right)$ then $Q(t)$ converges to $Q_{\text {min }}$; otherwise $Q(t)$ leaves the interval $\left(R_{L}, R_{H}\right)$. When outside of this interval, it starts moving towards $\bar{Q}$, but not necessary converging to it. In fact, when starting from 
the initial state $C_{1}, Q(t)$ will move towards $\frac{S_{2}(1)}{\bar{S}_{1}}$, and when starting from the initial state $C_{2}, Q(t)$ will move towards $\frac{\bar{S}_{2}}{S_{1}(1)}$.

When starting from $C_{1}$,if $\frac{S_{2}(1)}{\bar{S}_{1}}<R_{L}$, the system will remain in $C_{1}$ indefinitely. If $R_{L}<\frac{S_{2}(1)}{\bar{S}_{1}}<R_{H}$, the system will enter the state $C_{3}$. If $\frac{S_{2}(1)}{\bar{S}_{1}}>R_{H}$, weather the system enters the state $C_{3}$ or not after a certain number of steps will depend on the additional condition (**) from the bottom of the Table A.4, which can be derived using elementary algebra.

Similar reasoning can be applied for system starting in $C_{2}$.

Weather the system remains in state $C_{3}$ or not depends on the condition $Q_{\mathrm{m} \mathrm{in}} \in\left(R_{L}, R_{H}\right)$.

If we start alternating between the two states, we either forever alternate between states $C_{1}$ and $C_{3}$, end up in state $C_{1}$ or end up in state $C_{2}$. Ending in state $C_{2}$ is only possible if $\bar{Q}>R_{H}$, and can only happen if condition $(* * *)$ is satisfied, derived similarly to conditions (*) and (**). Otherwise, if $\frac{S_{L}}{\bar{S}_{1}}<R_{L}$, we will stabilize in $C_{1}$, and if $\frac{S_{L}}{\bar{S}_{1}}>R_{L}$ we will alternate between states indefinitely. Namely, if the system stabilizes in $C_{1}$, then $S_{1}(t)$ will converge to $\bar{S}_{1}$, while at any time certainly $S_{2}(t) \geq S_{L}$. Since in $C_{1}$ the value of $S_{2}(t)$ does not change and $S_{1}(t)$ converges to $\bar{S}_{1}$, we get $\frac{S_{L}}{\bar{S}_{1}} \leq R_{L}$. On the other side, when the system starts alternating between the two states, $S_{2}(t)$ will only change when the system is in state $C_{3}$, decreasing towards $S_{L}$. If the system alternates between the two states indefinitely, $S_{2}(t)$ will in fact converge to $S_{L}$. So, eventually $S_{2}(t)$ will be less than $\bar{S}_{2}$, and hence by Proposition 5.1, $S_{1}(t)$ will be greater or equal to $\overline{S_{1}}$. But then for large enough $t, Q(t)>R_{L}$ contradicts the condition $\frac{S_{L}}{\bar{S}_{1}}<R_{L}$, proving that this is also a sufficient condition for system to stabilize in $C_{1}$.

Putting these conditions together we obtain the desired result. 